\title{
Interactions of Alectinib with Human ATP-Binding Cassette Drug Efflux Transporters and Cytochrome P450 Biotransformation Enzymes: Effect on Pharmacokinetic Multidrug Resistance
}

\author{
(1) Jakub Hofman, Ales Sorf, @Dimitrios Vagiannis, Simona Sucha, Eva Novotna, \\ (1) Sarah Kammerer, Jan-Heiner Küpper, (1) Martina Ceckova, and 다rantisek Staud
}

Departments of Pharmacology and Toxicology (J.H., A.S., D.V., S.S., M.C., F.S.) and Biochemical Sciences (E.N.), Faculty of Pharmacy in Hradec Kralove, Charles University, Hradec Kralove, Czech Republic; and Institute of Biotechnology, Brandenburg University of Technology Cottbus-Senftenberg, Senftenberg, Germany (S.K., J.-H.K.)

Received February 26, 2019; accepted April 30, 2019

\begin{abstract}
Alectinib is a tyrosine kinase inhibitor currently used as a first-line treatment of anaplastic lymphoma kinase-positive metastatic nonsmall cell lung cancer (NSCLC). In the present work, we investigated possible interactions of this novel drug with ATP-binding cassette (ABC) drug efflux transporters and cytochrome P450 (P450) biotransformation enzymes that play significant roles in the phenomenon of multidrug resistance (MDR) of cancer cells as well as in pharmacokinetic drug-drug interactions. Using accumulation studies in Madin-Darby canine kidney subtype 2 (MDCKII) cells alectinib was identified as an inhibitor of $A B C B 1$ and $A B C G 2$ but not of $A B C C 1$. In subsequent drug combination studies, we demonstrated the ability for alectinib to effectively overcome MDR in ABCB1- and ABCG2-overexpressing MDCKII and A431 cells. To describe the
\end{abstract}

pharmacokinetic interaction profile of alectinib in a complete fashion, its possible inhibitory properties toward clinically relevant P450 enzymes (i.e., CYP1A2, CYP2B6, CYP2C8, CYP2C9, CYP2C19, CYP2D6, CYP3A4, or CYP3A5) were evaluated using human P450expressing insect microsomes, revealing alectinib as a poor interactor. Advantageously for its use in pharmacotherapy, alectinib further exhibited negligible potential to cause any changes in expression of $A B C B 1, A B C G 2, A B C C 1, C Y P 1 A 2, C Y P 3 A 4$, and CYP2B6 in intestine, liver, and NSCLC models. Our in vitro observations might serve as a valuable foundation for future in vivo studies that could support the rationale for our conclusions and possibly enable providing more efficient and safer therapy to many oncological patients.

\section{Introduction}

Globally, cancer is listed as the second major cause of death and is expected to surpass cardiovascular diseases in the next few years (Bhatnagar et al., 2015). Among cancerous diseases, lung cancer is far and away the leading killer and is responsible for approximately $25 \%$ of

This work was supported by the Czech Science Foundation [Grant 16-26849S], the Grant Agency of Charles University [Project 1568218/C], the Project EFSACDN (Efficiency and safety improvement of current drugs and nutraceuticals: advanced methods - new challenges) [CZ.02.1.01/0.0/0.0/16_019/0000841] cofunded by ERDF (European Regional Development Fund), and finally by Charles University [SVV/2019/260-414 and SVV/2019/260-416]. This work was further supported by the European Funds for Regional Development, Brandenburg, Germany, under the project "Entwicklung eines physiologisch relevanten Testsystems zur In-vitro-Erfassung von Hepatotoxizität im Hochdurchsatz" [85009748]. Computational resources were provided by the CESNET (Czech Education and Scientific NETwork) [LM2015042] and CERIT (Centre for Education, Research and Innovation for ICT) Scientific Cloud [LM2015085], under the program "Projects of Large Research, Development, and Innovations Infrastructures."

https://doi.org/10.1124/dmd.119.086975. all cancer deaths in both men and women (Siegel et al., 2018). Clinical treatment of tumor diseases is today moving from classic cytotoxic agents to novel targeted drugs that are characterized by high efficacy and reduced toxicity (DeVita and Chu, 2008). Alectinib (Alecensa) (Fig. 1) is a small molecule tyrosine kinase inhibitor (TKI) targeting fusion mutated anaplastic lymphoma kinase (ALK), which acts as an oncogenic driver in nonsmall cell lung cancer (NSCLC). This molecule gained accelerated approval by the US Food and Drug Administration in 2015 as a second-line treatment of patients with advanced ALK-positive NSCLC. Two years later, alectinib replaced crizotinib (Xalkori) as the first-line treatment of ALK-positive metastatic NSCLC both in the United States and Europe (Muller et al., 2017), thus becoming an essential drug against this type of cancer.

ATP-binding cassette (ABC) drug efflux transporters comprise an important group of transmembrane proteins that are able to pump a variety of structurally unrelated xenobiotic compounds, including drugs, out of cells. These essential carriers are predominantly localized in organs with absorptive and eliminative functions (intestine, liver, and kidney), as well as in body barriers (brain, testes, and placenta). According to their localizations, ABC drug efflux transporters act as body protection units limiting absorption and

ABBREVIATIONS: ABC, ATP-binding cassette; ALK, anaplastic lymphoma kinase; $\mathrm{Cl}$, combination index; DDI, drug-drug interaction; EMA, European Medicines Agency; $F_{A}$, fraction of cells affected; MDCKII, Madin-Darby canine kidney subtype 2; MDCKII-par, Madin-Darby canine kidney subtype 2 parent; MDR, multidrug resistance; M-site, modulator site; MTT, 3-(4,5-dimethyl-2-thiazolyl)-2,5-diphenyl-2H-tetrazolium bromide; NBD, nucleotide-binding domain; NSCLC, nonsmall cell lung cancer; P450, cytochrome P450; PCR, polymerase chain reaction; PDB ID, Protein Data Bank identification; R-site, rhodamine-binding site; TKI, tyrosine kinase inhibitor. 


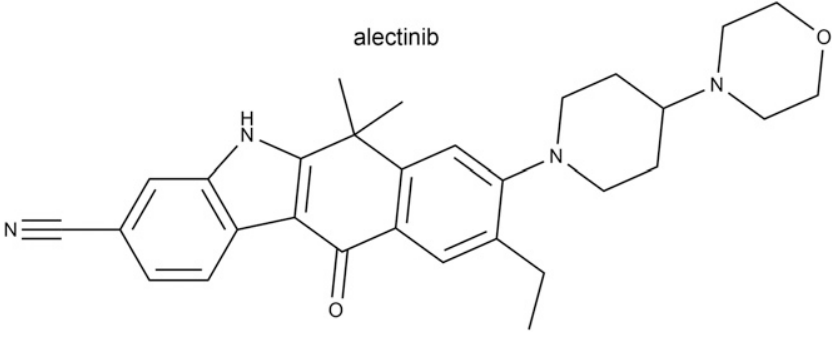

Fig. 1. Chemical structure of alectinib.

distribution while enhancing elimination of potentially harmful drugs. Such actions significantly affect the overall pharmacokinetic behavior of transporter substrates and create a rationale for clinically relevant pharmacokinetic drug-drug interactions (DDIs) (Szakács et al., 2008). The cytochrome P450 (P450) superfamily constitutes another crucial part of the body's detoxification capability and often cooperates with $\mathrm{ABC}$ drug efflux transporters in body protection. These biotransformation enzymes metabolize a broad spectrum of drugs, thus enhancing their excretion. Similarly to ABC transporters, P450s constitute an important site for pharmacokinetic DDIs (Wienkers and Heath, 2005). Therefore, investigation of novel chemical entities' interactions with $\mathrm{ABC}$ transporters and $\mathrm{P} 450$ enzymes is strongly highlighted by drug regulatory authorities, which also recommend the use of in vitro methods for assessing interaction potential (Prueksaritanont et al., 2013). Although the pharmacodynamic properties of alectinib have been studied extensively, only limited information is available regarding its pharmacokinetic behavior, and especially its interactions with $\mathrm{ABC}$ drug efflux transporters and P450 enzymes.

In addition to their beneficial protective functions in physiologic tissues, some of the ABC drug efflux transporters and P450 metabolizing enzymes expressed in tumor cells have been reported to reduce the intracellular concentrations of the active forms of anticancer drugs below their cytotoxic levels. Among ABC transporters, ABCB1, ABCG2, and $\mathrm{ABCC} 1$ have been shown to participate in multidrug resistance (MDR) in vitro and in vivo (Fletcher et al., 2010; Robey et al., 2018). In comparison with transporters, much less attention has been given to the role of metabolizing enzymes in MDR. Nevertheless, decreased efficacy of paclitaxel, docetaxel, and vincristine has been clearly linked to the activities of CYP3A4/5 and CYP2C8 enzymes in tumors (Michael and Doherty, 2005; Rochat, 2009; Vadlapatla et al., 2013). Several attempts have been made to overcome ABC transportermediated resistance by coadministration of $\mathrm{ABC}$ transporter inhibitors with conventional cytotoxic drugs, but these compounds have failed in clinical trials due to insufficient efficacy and/or toxicity (Bugde et al., 2017). Apart from this strategy, targeting pharmacokinetic MDR mechanisms still has appealing potential. In particular, combining new-generation modulators identified among novel small molecule targeted drugs with conventional anticancer drugs has potential to become a new treatment option for MDR cancers (Kathawala et al., 2015; Beretta et al., 2017). We have recently revealed inhibitory effects of several cyclin-dependent kinase inhibitors in relation to $\mathrm{ABCB} 1, \mathrm{ABCG} 2$, and/or $\mathrm{ABCC} 1$, as well as their abilities to reverse MDR of $\mathrm{ABC}$ transporter-expressing cells toward conventional cytotoxic anticancer drugs (Hofman et al., 2012; Cihalova et al., 2013, 2015a,b; Sorf et al., 2018). This dual mechanism could be proven advantageous and may imply a valuable role for these drugs in various chemotherapeutic regimens.

In the present work, we used a variety of in vitro techniques to investigate the potential for alectinib to become the perpetrator of DDIs mediated by ABC drug efflux transporters and/or P450s. Furthermore, we tested the role of alectinib in pharmacokinetic MDR and its capability to overcome this unfavorable therapeutic obstacle.

\section{Materials and Methods}

Reagents and Chemicals. Alectinib was obtained from Selleckchem (Houston, TX). Hoechst 33342 and calcein AM were obtained from Sigma Aldrich (St. Louis, MO) and Thermo Fisher Scientific (Waltham, MA), respectively. Daunorubicin, mitoxantrone, DMSO, 3-(4,5-dimethyl-2thiazolyl)-2,5-diphenyl-2 $H$-tetrazolium bromide (MTT), as well as cell culture reagents were purchased from Sigma Aldrich. Opti-MEM was supplied by Gibco BRL Life Technologies (Rockville, MD). Hepatocyte Culture Medium and Hepatocyte High Performance Medium were obtained from Upcyte Technologies (Hamburg, Germany). LY335979 [(2R)-1-\{4[(1aR,10bS)-1,1-Difluoro-1,1a,6,10b-tetrahydrodibenzo[a,e]cyclopropa[c][7] annulen-6-yl]piperazin-1-yl\}-3-(quinolin-5-yloxy)propan-2-ol] (zosuquidar) was obtained from Toronto Research Chemicals (North York, ON, Canada), and Ko143 [(3S,6S,12aS)-1,2,3,4,6,7,12,12a-Octahydro-9-methoxy-6-(2-methylpropyl)1,4-dioxopyrazino $\left[1^{\prime}, 2^{\prime}: 1,6\right]$ pyrido[3,4-b]indole-3-propanoic acid 1,1-dimethylethyl ester] and MK-571 (3-[[3-[(E)-2-(7-chloroquinolin-2-yl)ethenyl]phenyl](3-dimethylamino-3-oxopropyl)sulfanylmethyl]sulfanylpropanoic acid) were obtained from Enzo Life Sciences (Farmingdale, NY). Insect cell microsomes containing recombinant human enzymes CYP1A2, CYP2B6, CYP2C8, CYP2C9, CYP2C19, CYP2D6, CYP3A4, or CYP3A5 were purchased as Vivid CYP450 Screening Kits from Thermo Fisher Scientific. P450 inhibitors $\alpha$-naphthoflavone (CYP1A2), miconazole (CYP2B6 and CYP2C19), montelukast (CYP2C8), sulfaphenazole (CYP2C9), quinidine (CYP2D6), and ketoconazole (CYP3A4 and CYP3A5) were obtained from Sigma Aldrich. Pierce BCA Protein Assay Kit was purchased from Thermo Fisher Scientific. TRI Reagent was purchased from Molecular Research Center (Cincinnati, $\mathrm{OH})$. TaqMan systems for the analyses of $A B C B 1, A B C G 2, A B C C 1, C Y P 1 A 2$, $C Y P 3 A 4$, and $C Y P 2 B 6$ mRNA expressions, gb Reverse Transcription Kit, and gb Easy PCR Master Mix were obtained from Generi Biotech (Hradec Kralove, Czech Republic). All other chemicals and reagents were of the highest purity commercially available.

Cell Cultures. Madin-Darby canine kidney subtype 2 (MDCKII) cell lines transduced for stable expression of human transporters $\mathrm{ABCB} 1$ (MDCKIIABCB1), ABCG2 (MDCKII-ABCG2), or ABCC1 (MDCKII-ABCC1) and the control MDCKII-parent (MDCKII-par) cell line were all obtained from Dr. Alfred Schinkel (The Netherlands Cancer Institute, Amsterdam, The Netherlands). Human epidermoid carcinoma A431-parent cells and their $\mathrm{ABCB} 1-$ and $\mathrm{ABCG} 2$-overexpressing variants $\mathrm{A} 431-\mathrm{ABCB} 1$ and $\mathrm{A} 431-$ ABCG2, respectively, were kindly provided by Dr. Balasz Sarkadi (Hungarian Academy of Sciences, Budapest, Hungary) (Elkind et al., 2005). The MDCKII and A431 cells were grown in high-glucose Dulbecco's modified Eagle's medium supplemented with 10\% FBS. A549 (human NSCLC adenocarcinoma) cells were purchased from American Type Culture Collection (Manassas, VA) and cultured under identical conditions as the MDCKII sublines. Caco-2 (human colorectal adenocarcinoma) and NCI-H1299 (human NSCLC carcinoma) cell lines were purchased from the same source as A549 and were cultured in high-glucose Dulbecco's modified Eagle's medium supplemented with $10 \%$ FBS along with $1 \%$ nonessential amino acids and RPMI 1640 supplemented with $10 \%$ FBS, 1 mM sodium pyruvate, and $10 \mathrm{mM}$ HEPES. LS174T (human colorectal adenocarcinoma) cells were obtained from the European Collection of Cell Cultures (Salisbury, UK) and cultured in Eagle's minimal essential medium supplemented with $2 \mathrm{mM}$ glutamine, $1 \%$ nonessential amino acids, and $10 \%$ FBS. The unique model of proliferation-competent primary-like human hepatocytes HepaFH3 was kindly provided by Professor Jan-Heiner Küpper (Brandenburgische Technische Universität, Cottbus-Senftenberg, Germany) (Herzog et al., 2016). These cells were cultured in complete Hepatocyte Culture Medium, and for experiments complete Hepatocyte High Performance Medium was used. Routine cultivations and all experiments were performed under standard conditions $\left(37^{\circ} \mathrm{C}\right.$ and $\left.5 \% \mathrm{CO}_{2}\right)$ in antibiotic-free medium. The cell lines were periodically tested for mycoplasma contamination. Cells from passages 10-25 were used in all experiments. DMSO was applied as a solvent for alectinib at concentrations that did not exceed $0.5 \%$. No effects on the tested parameters were observed in the control experiments. 
Cellular Accumulation Assay with Hoechst 33342 and Calcein AM. In brief, MDCKII-par, MDCKII-ABCB1, MDCKII-ABCG2, and MDCKII$\mathrm{ABCC} 1$ were seeded at densities of 5.0, 5.0, 5.5, and $6.0 \times 10^{4}$ cells/well on transparent 96 -well plates 24 hours before the experiment and incubated at $37^{\circ} \mathrm{C}$ in $5 \% \mathrm{CO}_{2}$ to grow to full confluence. Cells were then washed twice with $1 \mathrm{X}$ PBS, Opti-MEM solutions of alectinib $(0.1,0.5,1,2.5$, and $5 \mu \mathrm{M})$ and model

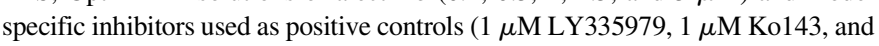
$25 \mu \mathrm{M}$ MK-571 for ABCB1, ABCG2, and ABCC1, respectively) were added, and plates were preincubated for 10 minutes under identical conditions. Hoechst 33342 (model substrate for $\mathrm{ABCB} 1$ and $\mathrm{ABCG}$ ) or calcein $\mathrm{AM}$ (model substrate for $\mathrm{ABCC} 1$ ) in Opti-MEM at a final concentration of 8 or $2 \mu \mathrm{M}$, respectively, was added rapidly into all wells except blank samples and their intracellular levels were determined at 1-minute intervals for 30 minutes using an Infinite M200 Pro microplate reader (Tecan, Männedorf, Switzerland). Fluorescence was monitored in a bottom mode using excitation/emission wavelengths of 350/465 and 485/535 nm for Hoechst 33342 and calcein AM, respectively.

Cellular Accumulation Assay with Daunorubicin and Mitoxantrone. MDCKII-par, MDCKII-ABCB1, MDCKII-ABCG2, and MDCKII-ABCC1 cells were seeded on a 12-well culture plate at a density of $1.5 \times 10^{5}$ cells/well and cultured for 24 hours to reach $70 \%-80 \%$ confluence. The cells were then washed once with 1X PBS, treated with Opti-MEM solutions of alectinib $(0.01$, $0.1,0.5,1,2.5$ and $5 \mu \mathrm{M})$ or model-specific inhibitors $(1 \mu \mathrm{M} \mathrm{LY} 335979,1 \mu \mathrm{M}$ Ko143, and $25 \mu \mathrm{M}$ MK-571 for ABCB1, ABCG2, and ABCC1, respectively), and preincubated for 10 minutes at $37^{\circ} \mathrm{C}$ and $5 \% \mathrm{CO}_{2}$. Afterward, daunorubicin (substrate for $\mathrm{ABCB} 1$ and $\mathrm{ABCC} 1$ ) or mitoxantrone (substrate for $\mathrm{ABCG} 2$ ) in Opti-MEM was added to the cells in the final concentration of 2 or $1 \mu \mathrm{M}$, respectively, with the exception of background samples. After 1-hour incubation under the same conditions, the cells were put on ice, washed twice with ice cold 1X PBS, and lysed in lysis buffer $(25 \mathrm{mM}$ Tris, $150 \mathrm{mM} \mathrm{NaCl}$, and $1 \%$ Triton $\mathrm{X}-100)$ on an automatic shaker for 30 minutes at room temperature. The lysate was resuspended, harvested into Eppendorf tubes, and subsequently centrifuged at $10,000 \mathrm{rpm}$ for 5 minutes. Next, $100 \mu \mathrm{l}$ of pure lysate free of cellular debris from each sample was transferred into black 96-well plates and fluorescence was measured using an Infinite M200 Pro microplate reader (Tecan). The excitatio$\mathrm{n} / \mathrm{emission}$ wavelengths for daunorubicin and mitoxantrone were 490/565 and $640 / 670 \mathrm{~nm}$, respectively. The protein contents of cell lysates were assessed via the bicinchoninic acid method employing a Pierce BCA Protein Assay Kit. Relative fluorescence unit values obtained in fluorescence measurements were normalized to protein content.

Molecular Docking Simulations. Alectinib was downloaded from the ZINC Database (http://zinc.docking.org) (Irwin et al., 2012) and its energy was minimized using CS ChemOffice version 18.0 (CambridgeSoft Corporation, Cambridge, MA). The structures of ABCB1 were obtained from the Protein Data Bank [http://www.rcsb.org; Protein Data Bank identifications (PDB IDs) 4M2S and 6COV] (Li et al., 2014; Kim and Chen, 2018). A homology model of the inward-facing form was generated using the crystal structure of mouse Abcb1 (PDB ID 4M2S) (Li et al., 2014) and primary sequence of human ABCB1 (P08183) (UniProt Consortium, 2019) by means of the SWISS-MODEL Workspace accessible via the ExPaSy web server (Guex et al., 2009; Bienert et al., 2017; Waterhouse et al., 2018). In the case of the outward-facing form, the SWISS-MODEL Workspace was employed to generate a homology model with Gln-556 and Gln-1201 mutated to catalytically active Glu using the same human $\mathrm{ABCB} 1$ sequence and $6 \mathrm{COV}$ as a template. Both alectinib and $\mathrm{ABCB} 1$ were further prepared for docking using MGL Tools 1.5.6 (Morris et al., 2009). In the case of protein, all water molecules, ATP, and ligands were removed, and hydrogens and Gasteiger charges were then added. Docking calculations were carried out with AutoDock Vina 1.1.2 (Trott and Olson, 2010). Rigid docking was performed using an $80 \times 80 \times 80$ grid box positioned into the internal cavity of the inward-facing form $(x=18.62, y=55.05, z=-0.82)$. Flexible docking was further performed into both inward- and outward-facing forms of ABCB1. Docking into the modulator site (M-site) of the inward-facing form involves nine flexible protein residues (Phe-303, Tyr-307, Ile-340, Phe-343, Gln-347, Gln-725, Phe-728, Phe-983, and Gln-990), and the size of the grid box was decreased to $20 \times 20 \times 20$. In the case of rhodamine-binding site (R-site) docking, five residues (Thr-240, Asp-241, Leu-244, Lys-826, and Phe-994) were assigned as flexible and a $40 \times 40 \times 40$ grid box was placed into the $\mathrm{R}$-site position $(x=9.83$, $y=83.03, z=17.98$ ). The flexible part of the outward-facing form of ABCB 1 contains nine residues (Asp-164, Tyr-401, Arg-404, Ile-409, Lys-433, Thr-435,
Gln-475, Gln-1175, and Gln-1180) for docking into nucleotide-binding domain (NBD) 1 and seven residues (Gln-530, Tyr-1044, Arg-1047, Val-1052, Lys-1076, His-1232, and Gln-1118) for docking into NBD2. The $40 \times 40 \times 40$ grid box was positioned into ATP-binding sites $(x=172.27, y=190.31, z=132.08$ and $x=156.69, y=168.89, z=119.33)$. The exhaustiveness parameter was set to 8 for all docking calculations. PyMOL 1.8.6.0 (The PyMOL Molecular Graphics System; Schrödinger, LLC) was employed to visualize the interactions.

The structures of ABCG2 downloaded from the Protein Data Bank (PDB IDs 6HIJ and 6HBU) (Jackson et al., 2018; Manolaridis et al., 2018) were prepared for docking using MGL Tools 1.5.6 as described for ABCB1. Because the crystal structure with PDB ID 6HBU had been deposited into the Protein Data Bank with mutation E211Q, the SWISS-MODEL Workspace was used to change Gln-211 in the NBDs back to Glu-211 utilizing the primary sequence of human ABCG2 (Q9UNQ0) (UniProt Consortium, 2019) and crystal structure 6HBU as a template. AutoDock Vina was further employed for docking using the same conditions (size of grid boxes and exhaustiveness) as described previously. Docking calculations were carried out in the ligand-binding internal cavity $(x=129.81, y=129.91, z=142.89$; PDB ID 6HIJ) and NBDs $(x=113.32$, $y=92.03, z=129.89$ and $x=94.09, y=115.37, z=129.96$; PDB ID 6HBU). Six residues were assigned as flexible (Phe-432, Phe-439, Leu-539, Ile-543, Val-546, and Met-549) in the cases of flexible docking analysis performed in the internal cavity.

Inhibitory Assay for Human Recombinant P450s. CYP3A4, CYP3A5, and CYP2C8, the enzymes participating in cytostatic drug resistance, as well as all other P450 enzymes recommended to be tested for inhibition by the US Food and Drug Administration and the European Medicines Agency (EMA) (i.e., CYP1A2, CYP2B6, CYP2C9, CYP2C19, and CYP2D6), were included in the evaluation. Inhibition was tested employing Vivid CYP450 Screening Kits that contain microsomal preparations from insect cells expressing particular recombinant human P450 enzymes. Experiments were performed according to the manufacturer's instructions in a kinetic mode. Time-dependent generation of fluorescent metabolite was recorded at 1-minute intervals for 60 minutes using an Infinite M200 Pro microplate reader (Tecan). The enzyme concentration and incubation interval used for data evaluation (15 minutes) were within the linear part of the appropriate reaction velocity curves. The final concentration of DMSO used as an alectinib solvent did not exceed $0.5 \%$, and activity changes caused by DMSO were corrected using appropriate controls. Model inhibitors recommended by the manufacturer were used as positive controls in all experiments.

MTT Proliferation Assay. Cells were seeded at the following densities (cells/well): $1.3 \times 10^{4}$ for MDCKII-par, MDCKII-ABCB1, MDCKII-ABCG2, and MDCKII-ABCC1; $1.2 \times 10^{4}$ for A431-parent and A431-ABCB1; $1.0 \times 10^{4}$ for A431-ABCG2; $0.80 \times 10^{4}$ for A549; $0.75 \times 10^{4}$ for NCI-H1299; $2.0 \times 10^{4}$ for Caco-2; $5.0 \times 10^{4}$ for LS174T; and $1.0 \times 10^{4}$ for HepaFH3 on 96-well culture plates and cultured for 24 hours to reach $50 \%$ confluence. The medium was then replaced with fresh medium containing the tested drugs or drug combinations. Cell viability was determined after 48 hours of incubation under standard conditions $\left(37^{\circ} \mathrm{C}\right.$ and $\left.5 \% \mathrm{CO}_{2}\right)$. The medium was aspirated and cells were washed once with prewarmed 1 X PBS. MTT solution in Opti-MEM $(1 \mathrm{mg} / \mathrm{ml})$ was added to the cells, and the cells were subsequently incubated under standard conditions for 60 minutes (MDCKII-cell lines and HepaFH3 cells) or 45 minutes (all other cell lines). The solution was aspirated and the cells were lysed with DMSO in an automatic shaker for 10 minutes. Absorbance was measured at 570 and $690 \mathrm{~nm}$ using an Infinite M200 Pro microplate reader (Tecan). The background values of $690 \mathrm{~nm}$ were subtracted from those values obtained at $570 \mathrm{~nm}$. For the normalization of absorbance values, absorbance from cells where only medium had been added was considered as $100 \%$ viability. Absorbance equal to $0 \%$ viability was obtained from cells incubated in $10 \%$ DMSO. The method was used to 1) assess the effect of ABCB1, ABCG2, or ABCC1 on sensitivity of MDCKII cells to alectinib, 2) assess alectinib's potential to reverse MDR in drug combination assays, and 3) estimate the concentrations to be used in induction studies.

Drug Combination Assays. MDCKII and A431 sublines were seeded (see the aforementioned densities) on 96-well culture plates and cultured for 24 hours to reach $50 \%$ confluence. The medium was then replaced with fresh medium containing concentration ranges of alectinib alone or of a model cytostatic (daunorubicin or mitoxantrone) with or without $2 \mu \mathrm{M}$ (daunorubicin combinations) or $1 \mu \mathrm{M}$ (mitoxantrone combinations) alectinib. Cell viability was determined after 48 hours of incubation under standard conditions $\left(37^{\circ} \mathrm{C}\right.$ 
and $5 \% \mathrm{CO}_{2}$ ) according to the procedure described previously. The obtained data were converted to the fraction of cells affected $\left(\mathrm{F}_{\mathrm{A}}\right)$ data and the drug combination effect was quantified according to the combination index (CI) method of Chou-Talalay using CompuSyn 3.0.1 software (ComboSyn Inc., Paramus, NJ). Based on the CI values, the effects of simultaneously administered drugs were marked as either synergistic $(\mathrm{CI}<0.9)$, additive $(\mathrm{CI}=0.9-1.1)$, or antagonistic $(\mathrm{CI}>1.1)$ (Chou, 2006).

Gene Induction Studies. First, a suitable concentration of alectinib for gene expression studies was chosen using the MTT proliferation method (as previously described). For gene induction studies, A549 $\left(24 \times 10^{4}\right.$ cells/well), NCI-H1299 $\left(18 \times 10^{4}\right.$ cells/well $)$, Caco-2 $\left(50 \times 10^{4}\right.$ cells/well $)$, LS174T $\left(100 \times 10^{4}\right.$ cells/well), or HepaFH3 $\left(40 \times 10^{4}\right.$ cells/well) cells were seeded on 12 -well plates 24 hours before the experiment. At time 0 , the medium was replaced with fresh medium containing $0.5 \mu \mathrm{M}$ alectinib or $0.005 \%$ DMSO (vehicle control). Samples were taken at 24- and 48-hour intervals. Total RNA from the cells was isolated using TRI Reagent and chloroform according to the manufacturer's instructions. After isolation, the RNA quality and integrity were verified by agarose gel electrophoresis. DNase treatment was not performed prior to reverse transcription because all TaqMan quantitative real-time reverse transcription polymerase chain reaction (PCR) systems were designed to span introns and/or cross intron/exon boundaries. Reverse transcription was conducted using a commercial gb Reverse Transcription Kit, and 1000 ng of RNA was transcribed into the corresponding cDNA in one reaction. The $A B C B 1, A B C G 2, A B C C 1$, $C Y P 1 A 2, C Y P 3 A 4$, and $C Y P 2 B 6$ mRNA levels were determined using commercial gb Easy PCR Master Mix and TaqMan quantitative real-time reverse transcription PCR systems in 384-well plates according to the manufacturer's instructions while amplifying $20 \mathrm{ng}$ of cDNA per reaction. Relative quantification of the examined $\mathrm{ABC}$ transporters and $\mathrm{P} 450$ enzymes was performed using the $\triangle \Delta \mathrm{Ct}$ method; the geometric mean of B2M and HPRTI levels was used as an internal control to normalize the variability in expression levels. Quantitative realtime reverse transcription PCR analysis was performed on a QuantStudio 6 apparatus (Life Technologies, Carlsbad, CA) with initial denaturation at $95^{\circ} \mathrm{C}$ for 3 minutes and 40 repeats of a cycle consisting of $95^{\circ} \mathrm{C}$ for 10 seconds and $60^{\circ} \mathrm{C}$ for 20 seconds.

Statistical Analysis. Statistical analysis was performed using GraphPad Prism software version 7.03 (GraphPad Software Inc., La Jolla, CA). The $P$ values were calculated using one-way ANOVA followed by Dunnett's post hoc test or a twotailed unpaired $t$ test. Differences of $P<0.05$ were considered statistically significant. GraphPad Prism software was also used to calculate the $\mathrm{IC}_{50}$ values while applying nonlinear regression analysis using sigmoidal Hill kinetics (ABC transporter and $\mathrm{P} 450$ inhibitory assays and proliferation experiments) and to normalize the raw data ( $\mathrm{P} 450$ inhibitory assays and proliferation experiments). All experiments were performed as three independent repetitions with biologic triplicates in each repetition.

\section{Results}

Effect of Alectinib on Intracellular Accumulation of Hoechst 33342 or Calcein AM in MDCKII Sublines. First, alectinib's ability to inhibit ABC transporter-mediated efflux of Hoechst 33342 or calcein $\mathrm{AM}$ was investigated in MDCKII sublines. Alectinib significantly increased Hoechst 33342 accumulation in both MDCKII-ABCB1 and MDCKII-ABCG2 cells, reaching inhibitory potency of model inhibitor only in MDCKII-ABCG2 cells. Data analysis revealed relative $\mathrm{IC}_{50}$ values of 0.399 and $>5.00 \mu \mathrm{M}$ for ABCG2 and ABCB1 transporter inhibition, respectively. In contrast, no inhibitory effect of alectinib on ABCC1-mediated efflux of calcein AM was observed (Fig. 2).

Influence of Alectinib on Intracellular Accumulation of Daunorubicin or Mitoxantrone in MDCKII Sublines. To confirm the results obtained in experiments with Hoechst 33342 and calcein AM, we subsequently performed transporter inhibitory studies using daunorubicin (ABCB1 and $\mathrm{ABCC} 1$ substrates) and mitoxantrone (ABCG2 substrate). At the same time, these second-line accumulation experiments clarified alectinib to be applicable as an MDR modulator inasmuch as the same drugs were further employed as model agents in follow-up drug combination studies.
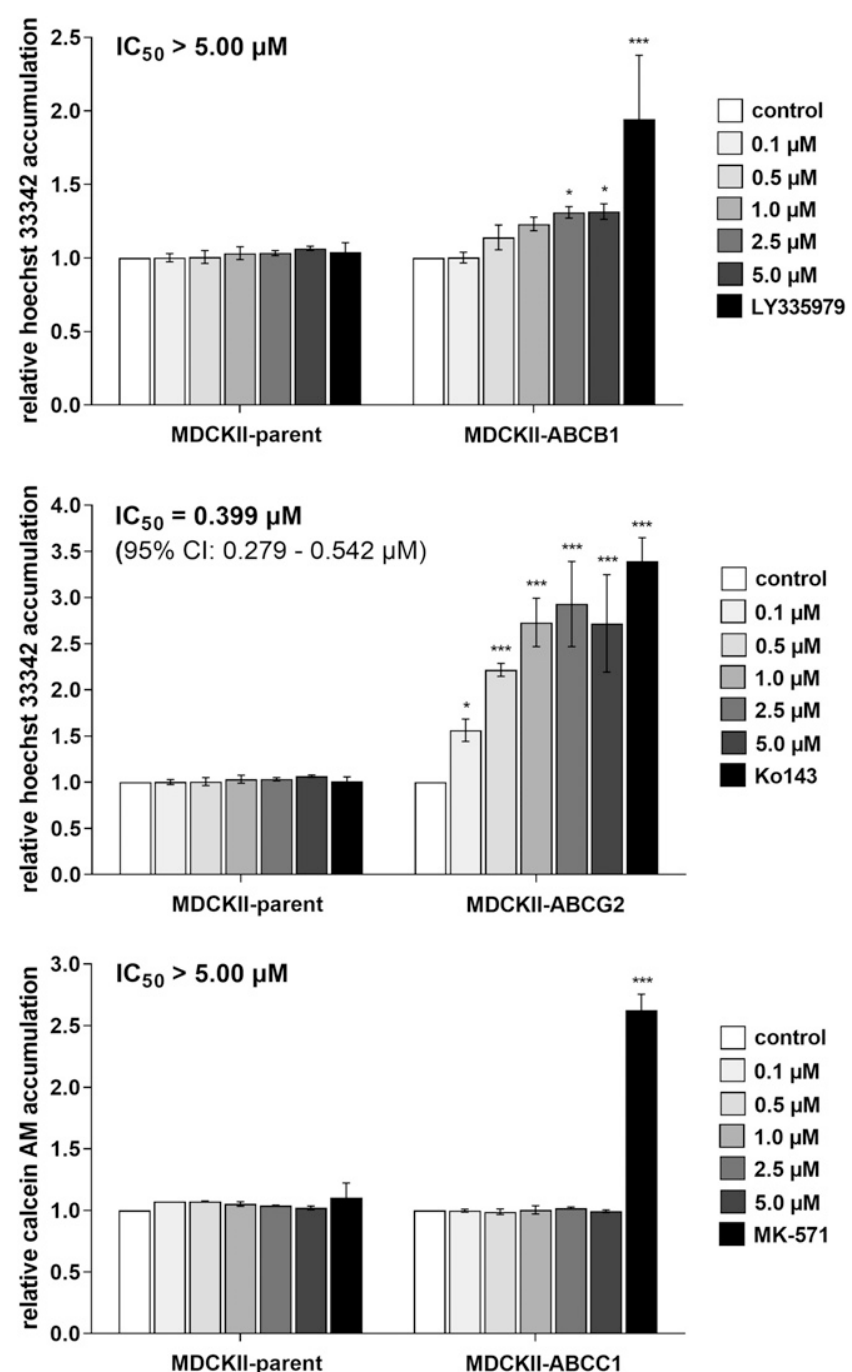

Fig. 2. Effects of alectinib on intracellular accumulation of Hoechst 33342 or calcein AM in MDCKII-par, MDCKII-ABCB1, MDCKII-ABCG2, and MDCKIIABCC1 cells. LY335979 $(1 \mu \mathrm{M})$, Ko143 $(1 \mu \mathrm{M})$, and MK-571 $(25 \mu \mathrm{M})$ were used as model inhibitors for the respective transporters. Tested concentrations had been confirmed in our previous studies to yield maximal transporter inhibition. Cells were preincubated with alectinib or model inhibitors for 10 minutes, and then $8 \mu \mathrm{M}$ Hoechst 3342 or $2 \mu \mathrm{M}$ calcein AM was added and fluorescence was immediately monitored in bottom kinetic mode for 30 minutes. Data are presented as mean \pm S.D. obtained from three independent experiments. The $\mathrm{IC}_{50}$ values were determined as relative ones, in which the $100 \%$ inhibitory effect was attributed to the alectinib concentration showing the most effective response. Statistical analysis was performed using one-way ANOVA followed by Dunnett's post hoc test $\left({ }^{*} P<0.05\right.$; $* * * P<0.001$ compared with control).

As in the previous case, alectinib significantly increased accumulation of model substrates in both MDCKII-ABCB1 and MDCKII-ABCG2 cells, yielding $\mathrm{IC}_{50}$ values of 1.27 and $0.453 \mu \mathrm{M}$, respectively. Similar to the results of the calcein AM assay, alectinib did not affect daunorubicin accumulation in MDCKII-ABCC1 cells even at the highest concentration tested (Fig. 3).

Molecular Docking of Alectinib into ABCB1 and ABCG2. To investigate the observed alectinib interaction with $\mathrm{ABCB} 1$ and ABCG2 at the molecular level, in silico docking simulations were performed. Rigid molecular docking into the inward-facing form of ABCB1 predicted two potential binding sites for alectinib: one close to the apex of the internal V-shaped cavity $(-10.2$ to $-8.9 \mathrm{kcal} / \mathrm{mol})$ and another at a lower location $(-8.7 \mathrm{kcal} / \mathrm{mol})$, described previously as the $\mathrm{M}$-site and R-site, respectively (Ferreira et al., 2013). In contrast, no interaction was 

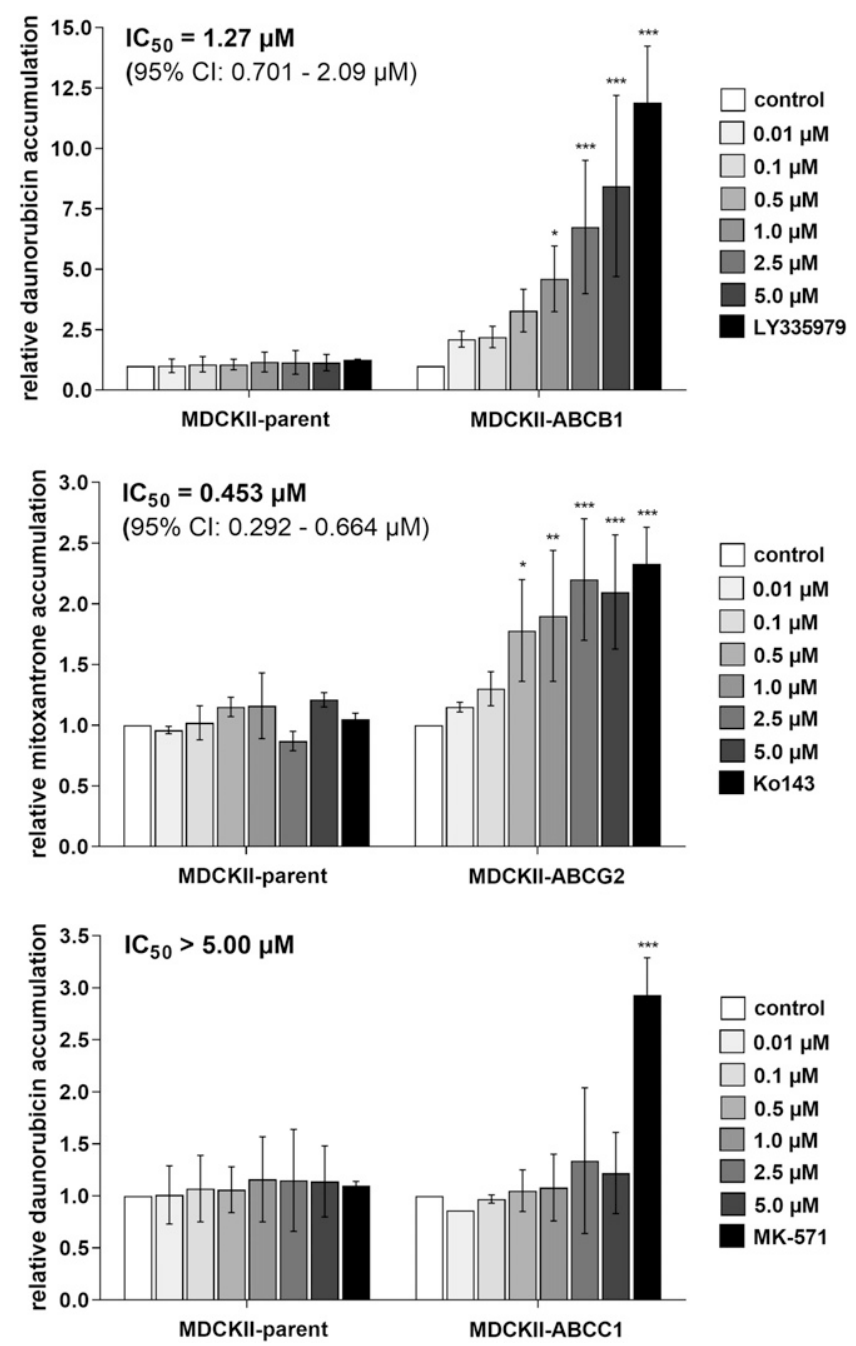

Fig. 3. Effects of alectinib on intracellular accumulation of daunorubicin or mitoxantrone in MDCKII-par, MDCKII-ABCB1, MDCKII-ABCG2, and MDCKIIABCC1 cells. LY335979 $(1 \mu \mathrm{M})$, Ko143 $(1 \mu \mathrm{M})$, and MK-571 $(25 \mu \mathrm{M})$ were used as model inhibitors for the respective transporters. The tested concentrations had been confirmed in our previous studies to yield maximal transporter inhibition. Cells were preincubated with alectinib or model inhibitors for 10 minutes, and then $2 \mu \mathrm{M}$ daunorubicin or $1 \mu \mathrm{M}$ mitoxantrone was added. After 1-hour incubation, cells were lysed and fluorescence was detected in lysates. Data are expressed as mean \pm S.D. obtained from three independent experiments. The $\mathrm{IC}_{50}$ values were determined as relative ones, in which the $100 \%$ inhibitory effect was attributed to the alectinib concentration showing the most effective response. Statistical analysis was performed using one-way ANOVA followed by Dunnett's post hoc test $(* P<0.05 ; * * P<0.01$; $* * * P<0.001$ compared with control).

observed on a third ligand-binding site specific for Hoechst 33342 (Fig. 4A). To mimic real conditions regarding possible $\mathrm{ABCB} 1$ conformations, the residues in the $\mathrm{M}$ - and R-sites predicted to interact with alectinib were set as flexible and molecular docking was repeated. The flexible docking analysis has shown that alectinib probably interacts with Met-69, Phe-303, Tyr-307, Phe-336, Ile-340, Phe-343, Gln725, Phe-728, Phe-732, Phe-983, Met-986, Gln-990, and Phe-994 (-12.5 $\mathrm{kcal} / \mathrm{mol}$ ) at the M-site and with Phe-770, Gln-773, Glu-782, Ala-823, Lys-826, Phe-994, and Pro-996 at the R-site (-9.4 kcal/mol) of ABCB1 (Fig. 4B). Because alectinib is an ATP-competitive inhibitor of its target ALK kinase (Song et al., 2015), we considered its potential interaction with NBDs of ABCB1 and docked alectinib at the ATP-binding site. The results of flexible molecular modeling show that alectinib may additionally compete with ATP $(-12.3$ and $-12.1 \mathrm{kcal} / \mathrm{mol})$, and thereby indirectly inhibit ABCB1 efflux function (Fig. 4C).
In contrast to $\mathrm{ABCB} 1, \mathrm{ABCG} 2$ is a homodimer that contains two cavities (internal and external) separated by Leu-554 of opposing monomers. However, only the internal cavity can bind ligands (Jackson et al., 2018). In our experiments, both rigid and flexible molecular docking positioned alectinib in the internal cavity, thereby showing that two molecules are necessary to lock ABCG2 into an inward-facing conformation (Fig. 4D). Further docking into NBDs of $\mathrm{ABCG} 2$ predicted that alectinib may probably compete with ATP for binding to NBDs $(-8.8$ and $-8.6 \mathrm{kcal} / \mathrm{mol})$ (Fig. 4E), albeit with significantly lower affinity than that predicted for the internal ligand binding cavity $(-13.1$ and $-13.0 \mathrm{kcal} / \mathrm{mol})$.

Modulatory Effects of Alectinib on ABC Transporter-Mediated Cytostatic MDR. In the follow-up experiments, we investigated whether inhibitory interactions of alectinib with $\mathrm{ABC}$ efflux transporters could be used to overcome resistance to the recognized ABCB1 and ABCG2 substrates daunorubicin and mitoxantrone, respectively. These studies were first performed in MDCKII sublines, and the results were then confirmed in a physiologically more relevant model of human cytostatic resistant A431 sublines (Elkind et al., 2005). The modulatory concentrations of alectinib ( 2 or $1 \mu \mathrm{M}$ for daunorubicin or mitoxantrone combinations, respectively) in the experiments were carefully selected to fit the following criteria: 1) causing sufficient inhibition of $\mathrm{ABC}$ transporter, 2) being only negligibly cytotoxic to the model cell lines, and 3 ) being clinically relevant based on the $C_{\max }$ values recorded in in vivo pharmacokinetic studies in patients.

Alectinib was shown to effectively overcome daunorubicin and mitoxantrone resistance in $\mathrm{ABC}$ transporter-overexpressing cells, yielding fold reversal values of 17.8 and 9.64 in MDCKII-ABCB1 and MDCKII-ABCG2 cells, respectively. The tested TKI was similarly active in A431 cells, reaching fold reversal values of 24.4 and 44.3 for $\mathrm{ABCB} 1$ - and $\mathrm{ABCG} 2$-overexpressing variant sublines, respectively. Although 1 or $2 \mu \mathrm{M}$ alectinib concentration caused only $7 \%$ or $11 \%$ and $6 \%$ or $24 \%$ viability decrease in MDCKII-par and A431-par cells, respectively, we observed unexpected sensitization of these control cells to daunorubicin's and mitoxantrone's effects. To subtract the nontransporter-mediated sensitization effect, we normalized fold reversal data from transporter-overexpressing cells to the fold reversal data from parent cells. The obtained normalized fold reversal values of $6.18,6.79,2.14$, and 4.81 in MDCKII-ABCB1, MDCKII-ABCG2, A431-ABCB1, and A431-ABCG2 cells, respectively, clearly reflect the participation of alectinib-caused $\mathrm{ABCB} 1$ and $\mathrm{ABCG} 2$ inhibitory effect in overcoming daunorubicin and mitoxantrone resistance (Fig. 5; Table 1). The involvement of inhibition of ABC efflux pumps in this process was further confirmed by employing data analysis according to the combination index method of Chou-Talalay, a method in wide use that offers quantitative definition for additive effect, synergism, or antagonism in drug combination studies. In addition to antagonistic and additive effects, synergism was also recorded in parent cells at a higher $\mathrm{F}_{\mathrm{A}}$ range, but its extent was considerably lower in comparison with $\mathrm{ABC}$ transporter-overexpressing cells (Fig. 6).

Effect of ABCB1, ABCG2, and ABCC1 Overexpression on Cell Resistance to Alectinib. In addition to evaluating the MDR-modulatory properties of alectinib, we also investigated whether ABC transporters could be the potential causative mechanism for developing cellular resistance to this TKI similar to standard cytostatics. The results of MTT proliferation studies showed no significant changes in alectinib antiproliferative effect between MDCKII-par and ABC transporter-transduced MDCKII sublines (Fig. 7), thus suggesting an improbable role for $\mathrm{ABCB} 1, \mathrm{ABCG} 2$, or $\mathrm{ABCC} 1$ efflux activity in the establishment of MDR to alectinib.

Alectinib Does Not Affect Activity of Clinically Relevant P450 Enzymes. To evaluate whether alectinib might have the potential to affect enzyme-mediated pharmacokinetic resistance to paclitaxel, docetaxel, or 
A

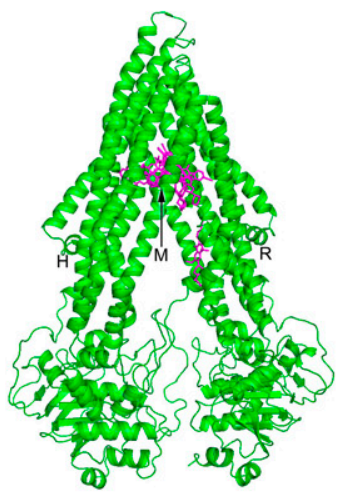

B

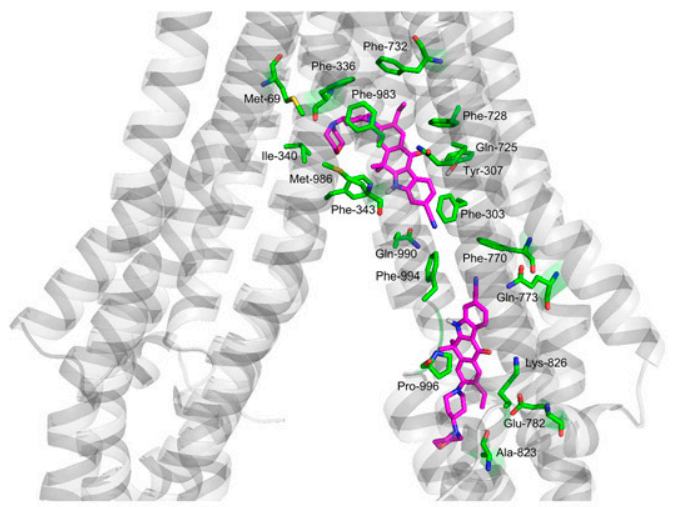

D
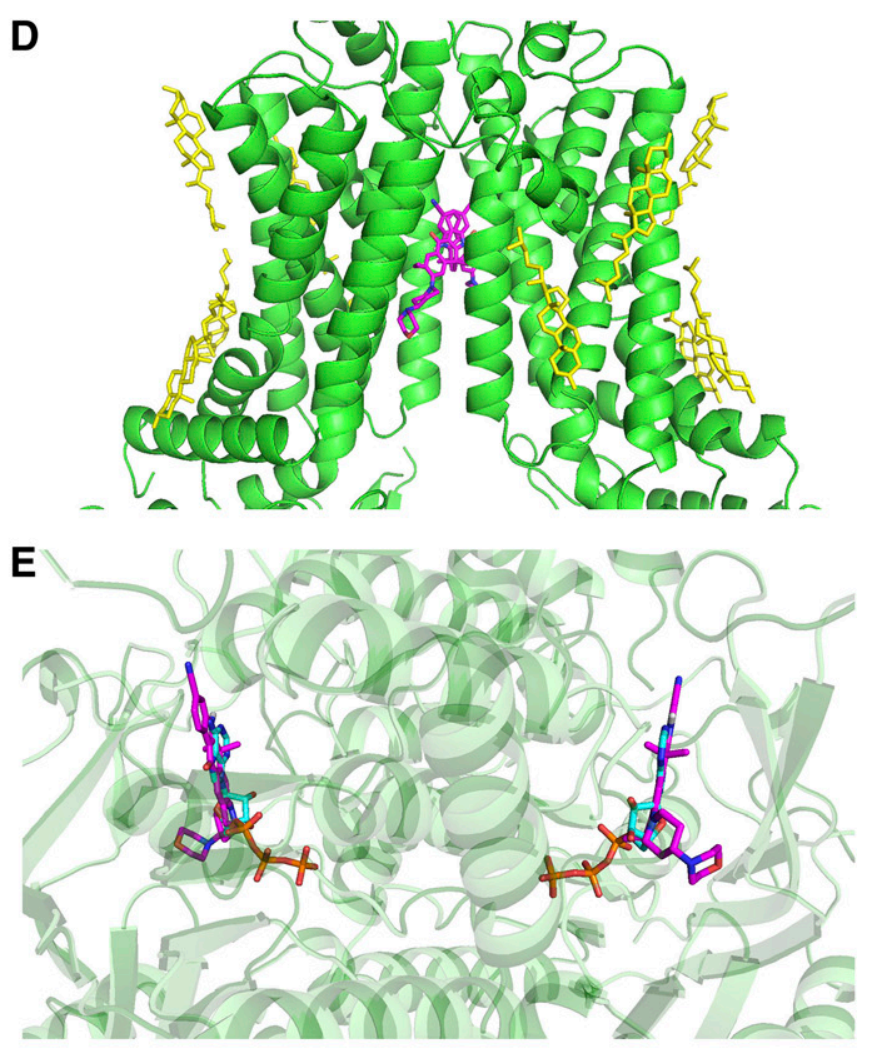

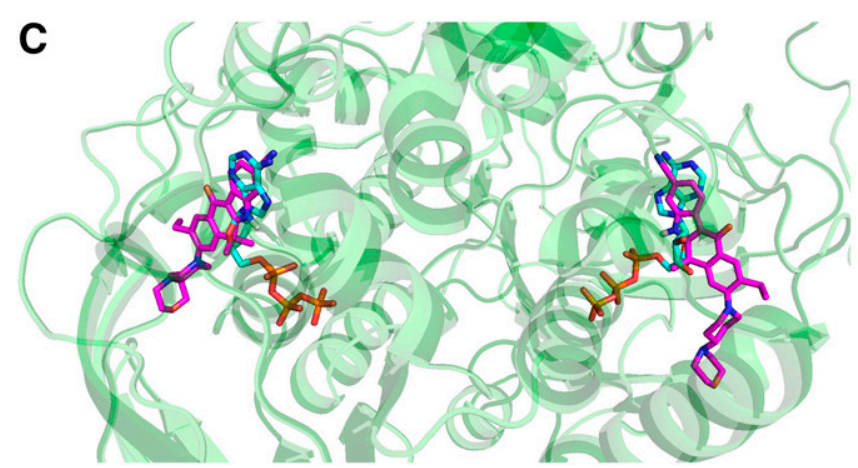

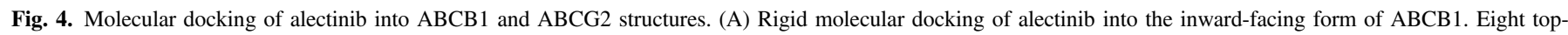

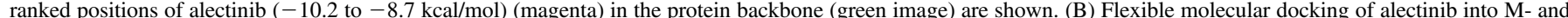

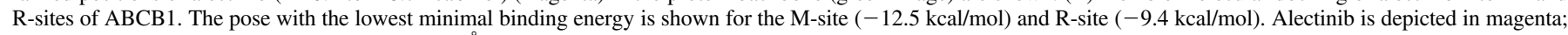

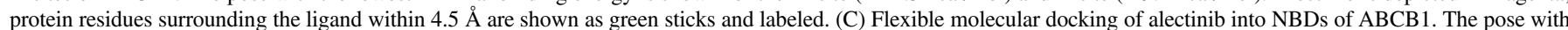

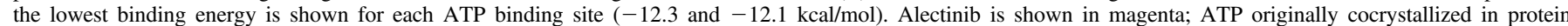

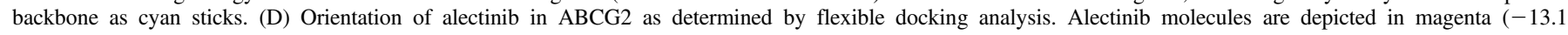

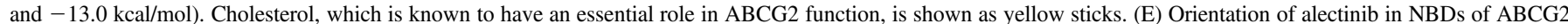
predicted by rigid docking analysis. Alectinib (magenta) may compete with ATP (cyan) for binding ( -8.8 and $-8.6 \mathrm{kcal} / \mathrm{mol}$ ) to NBDs.

vincristine, and become a perpetrator of DDIs, in vitro inhibitory assays with human recombinant CYP3A4, CYP3A5, and CYP2C8 were performed. The testing was further broadened to include other clinically relevant enzymes playing predominant roles in pharmacokinetic DDIs (i.e., CYP1A2, CYP2B6, CYP2C9, CYP2C19, and CYP2D6). Alectinib was demonstrated to be a poor interactor, since it did not reach an inhibitory level greater than $20 \%$ in any of these eight P450 enzymes even at the highest concentration tested (Fig. 8).

Changes in ABCB1, ABCG2, ABCC1, CYP1A2, CYP3A4, and CYP2B6 Expressions following Exposure to Alectinib. Apart from inhibition, the induction of $\mathrm{ABC}$ transporters and $\mathrm{P} 450$ s by alectinib could constitute another phenomenon that is able to affect the pharmacological fate of concomitantly administered drugs. To address this possibility, we performed gene expression studies focused on $A B C B 1, A B C G 2$, and $A B C C 1$ and $C Y P 1 A 2, C Y P 3 A 4$, and $C Y P 2 B 6$ in suitable intestine (LS174T and Caco-2) and hepatic (HepaFH3) cellular models, respectively. In addition, possible changes in $A B C B 1, A B C G 2$, and $A B C C 1$ gene expressions following exposure to alectinib were assessed in NSCLC cellular models (A549 and NCI-H1299) to reveal whether this TKI could affect the MDR phenotype of its target cancer cells.

The concentration of alectinib $(0.5 \mu \mathrm{M})$ in the experiments was carefully selected to fit the following criteria: 1) being only negligibly cytotoxic to model cell lines (Fig. 9A), and 2) being clinically relevant based on the $C_{\max }$ values recorded in in vivo pharmacokinetic studies of patients. Alectinib did not increase or decrease mRNA levels of the 

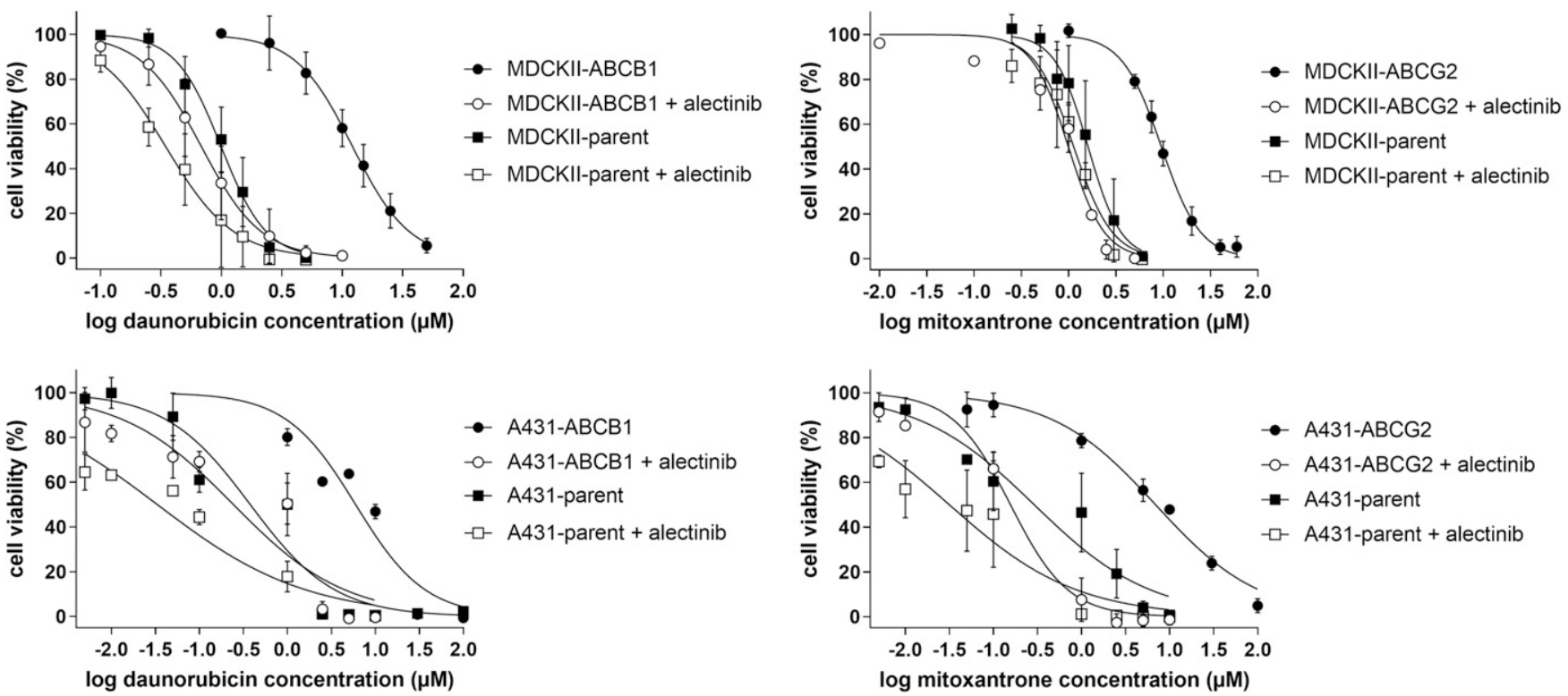

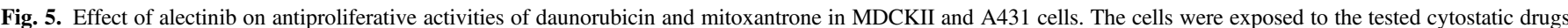

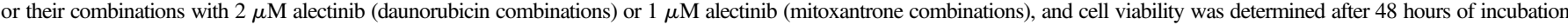
using the MTT proliferation test. The data obtained are further analyzed in Table 1 and Fig. 6 . Values are presented as mean \pm S.D. from three independent experiments.

investigated $\mathrm{ABC}$ transporters and $\mathrm{P} 450$ s by more than $100 \%$ or $50 \%$ in any of the cases (Fig. 9, B-F). Therefore, in accordance with EMA guidelines (European Medicines Agency, 2012), the results obtained cannot be considered positive for induction or downregulation. This means alectinib's potential to become a perpetrator of induction-based DDIs as well as to influence the MDR phenotype of its target cancer cells is low.

\section{Discussion}

Alectinib has become an essential drug for the treatment of patients suffering from fusion ALK-mutated forms of metastatic NSCLC (Muller et al., 2017). Although the pharmacodynamic properties of this drug have been well understood, its pharmacokinetic interactions with transport proteins and biotransformation enzymes remain to be elucidated. In the present work, we investigated alectinib interactions

TABLE 1

Analysis of the effect of simultaneous administration of alectinib with model cytotoxic drugs in MDCKII and A431 cells

\begin{tabular}{|c|c|c|c|c|c|}
\hline Cell line & Drug $^{a}$ & $\mathrm{IC}_{50}{ }^{b}$ & $95 \% \mathrm{CI}$ & Fold Reversal $^{c}$ & Normalized Fold Reversal $^{d}$ \\
\hline & & $\mu M$ & $\mu M$ & & \\
\hline \multicolumn{6}{|c|}{ MDCKII-parent } \\
\hline & Daunorubicin & 0.993 & $(0.867-1.13)$ & & \\
\hline & Mitoxantrone & 1.60 & $(1.43-1.81)$ & & \\
\hline & Daunorubicin + alectinib & $0.345^{*}$ & $(0.295-0.400)$ & 2.88 & \\
\hline & Mitoxantrone + alectinib & $1.13^{\mathrm{ns}}$ & $(0.909-1.40)$ & 1.42 & \\
\hline \multicolumn{6}{|c|}{ MDCKII-ABCB 1} \\
\hline & Daunorubicin & 12.1 & $(11.6-12.7)$ & & \\
\hline & Daunorubicin + alectinib & $0.681^{*}$ & $(0.650-0.714)$ & 17.8 & 6.18 \\
\hline \multicolumn{6}{|c|}{ MDCKII-ABCG2 } \\
\hline & Mitoxantrone & 9.55 & $(9.00-10.1)$ & & \\
\hline & Mitoxantrone + alectinib & $0.991 * *$ & $(0.707-1.29)$ & 9.64 & 6.79 \\
\hline \multicolumn{6}{|l|}{ A431-parent } \\
\hline & Daunorubicin & 0.378 & $(0.204-0.749)$ & & \\
\hline & Mitoxantrone & 0.290 & $(0.165-0.501)$ & & \\
\hline & Daunorubicin + alectinib & $0.0333^{\mathrm{ns}}$ & $(0.0211-0.0514)$ & 11.4 & \\
\hline & Mitoxantrone + alectinib & $0.0315^{\mathrm{ns}}$ & $(0.0152-0.0582)$ & 9.21 & \\
\hline \multicolumn{6}{|l|}{ A431-ABCB1 } \\
\hline & Daunorubicin + alectinib & $0.244 * * *$ & $(0.126-0.334)$ & 24.4 & 2.14 \\
\hline \multicolumn{6}{|l|}{ A431-ABCG2 } \\
\hline & Mitoxantrone & 6.91 & $(5.56-8.52)$ & & \\
\hline & Mitoxantrone + alectinib & $0.156^{* *}$ & $(0.111-0.220)$ & 44.3 & 4.81 \\
\hline
\end{tabular}

CI, confidence interval; ns, not significant.

${ }^{a}$ Alectinib in concentration of 2 or $1 \mu \mathrm{M}$ was used in daunorubicin and mitoxantrone combinations, respectively.

${ }^{b}$ Values were calculated from the data shown in Fig. 5. The $\mathrm{IC}_{50}$ values from drug combinations were compared with the $\mathrm{IC}_{50}$ values from single drug treatments in particular cell lines using two-tailed unpaired $t$ tests $(* P<0.05 ; * * P<0.01 ; * * * P<0.001)$.

${ }^{c}$ Fold reversal values were calculated as the ratio of the $\mathrm{IC}_{50}$ value from single drug treatment to the $\mathrm{IC}_{50}$ value of combined drug treatment in the particular cell line.

${ }^{d}$ Normalized fold reversal data represent the ratio of the $\mathrm{IC}_{50}$ value from combined drug treatment in transporter-overexpressing cells to the $\mathrm{IC}_{50}$ value from combined drug treatment in corresponding parent cells. 

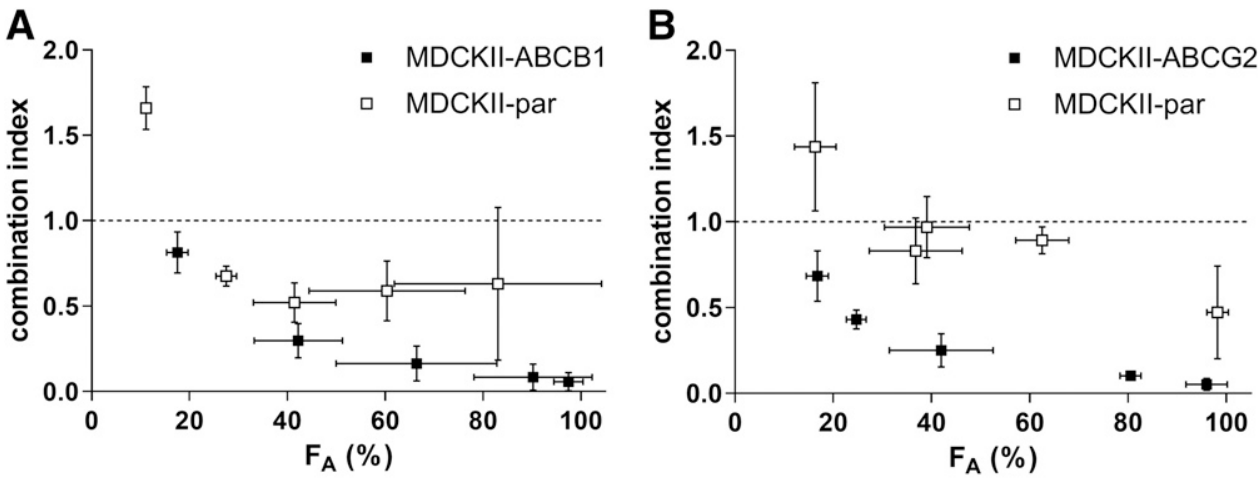

Fig. 6. Combination index analysis of the effects of 2 or $1 \mu \mathrm{M}$ alectinib on antiproliferative activities of daunorubicin or mitoxantrone, respectively, in MDCKII and A431 cells. Values shown in Fig. 5 together with alectinib proliferation data (not shown) were analyzed using CompuSyn software to obtain $\mathrm{Cl}$ plots. (A) alectinib + daunorubicin combination in MDCKII-ABCB1 cells, (B) alectinib + mitoxantrone combination in MDCKII-ABCG2 cells, (C) alectinib + daunorubicin combination in MDCKII-ABCB1 cells, and (D) alectinib +

C

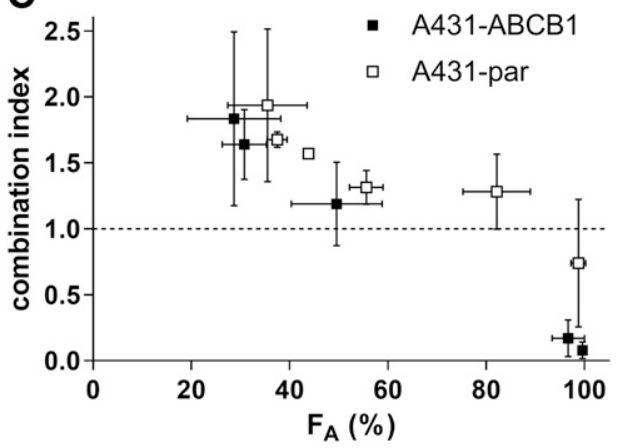

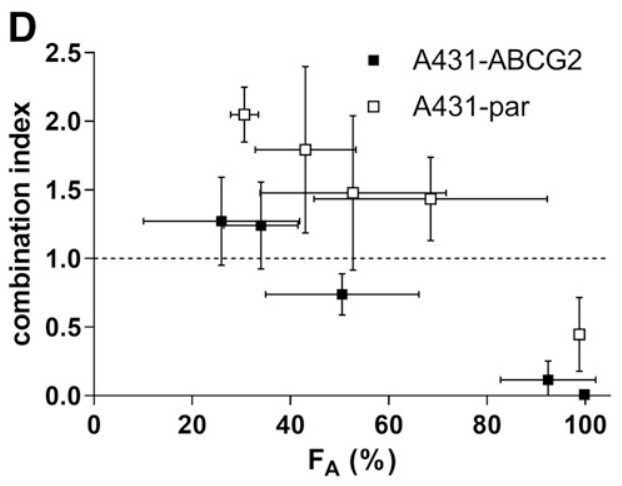
mitoxantrone combination in MDCKII-ABCG2 cells. According to the median-effect analysis principle developed by Chou-Talalay, CI values $<0.9$ determine the synergistic effect of drug combination, $\mathrm{CI}$ values $>0.9$ and $<1.1$ determine the additive effect, and $\mathrm{CI}$ values $>1.1$ determine the antagonistic effect. The data points are presented as mean \pm S.D. coming from three independent experiments.

with selected $\mathrm{ABC}$ drug efflux transporters and P450 enzymes and evaluated their possible utilization for overcoming pharmacokinetic MDR.

First, we investigated possible inhibition of $\mathrm{ABCB} 1, \mathrm{ABCG} 2$, and $\mathrm{ABCC} 1$ by alectinib in MDCKII sublines. While we obtained almost identical inhibitory characteristics toward $\mathrm{ABCG} 2$ and $\mathrm{ABCC} 1$ for both Hoechst 33342/calcein AM and daunorubicin/mitoxantrone, the extent of ABCB1 inhibition varied considerably between model substrates. Molecular docking revealed that alectinib binds to the M-site and R-site (specific for daunorubicin), but not to site specific for Hoechst 33342, thus explaining this discrepancy. It is noteworthy that our results highlight preferential affinity of substrates and inhibitors for any of the three $\mathrm{ABCB} 1$ binding sites as an important factor to consider when investigating and predicting $\mathrm{ABCB} 1-$ mediated drug-drug interactions. Both the interaction with substrate-binding sites and the competition with ATP observed in our docking studies are in accordance with recent reports demonstrating the competitive nature of $\mathrm{ABCB} 1$ inhibition by alectinib (Yang et al., 2017). Importantly, ABCB1 as well as the ABCG2 inhibitory activity of alectinib might be clinically relevant and worthy of further evaluations including in vivo studies inasmuch as the steady-state $C_{\max }$ value after maximum dose administration divided by the $\mathrm{IC}_{50}$ value as we assessed in vitro was substantially $>0.1$ (Giacomini et al., 2010). The alectinib steady-state $C_{\max }$ value assessed in clinical trials evaluating standard dosing of $600 \mathrm{mg}$ twice daily was equal to 1.38 $\mu \mathrm{M}$ (Zhu and $\mathrm{Ou}, 2017$ ).

Having confirmed $\mathrm{ABCB} 1$ and $\mathrm{ABCG} 2$ inhibition by alectinib, we hypothesized that these interactions could be favorably exploited for the modulation of MDR to cytostatic drugs, such as daunorubicin or mitoxantrone. To test this hypothesis, we combined these conventional anticancer drugs with alectinib in $\mathrm{ABC}$ transporter-overexpressing versus parental MDCKII and A431 cells and observed effective enhancement of their cytostatic effects in both cellular models. This is in accordance with a recent description of alectinib's ability to potentiate the anticancer effect of such various cytostatic drugs as paclitaxel or doxorubicin in several $\mathrm{ABC}$ transporter-overexpressing cell lines, leukemic xenografts, and primary leukemic cells (Yang et al., 2017). In addition to simple demonstration of the potential for alectinib to reverse MDR, in our studies we also focused on exact quantification of combination effects. In ABC transporter-overexpressing cells, synergism was detected as the outcome for the examined combinations across almost the whole range of $\mathrm{F}_{\mathrm{A}}$. This is an important finding, because synergistic combinations are frequently used in oncological clinical practice and allow for reducing the doses of drugs concomitantly used while dramatically increasing the safety and/or efficacy of a treatment (Bayat Mokhtari et al., 2017). Based on our

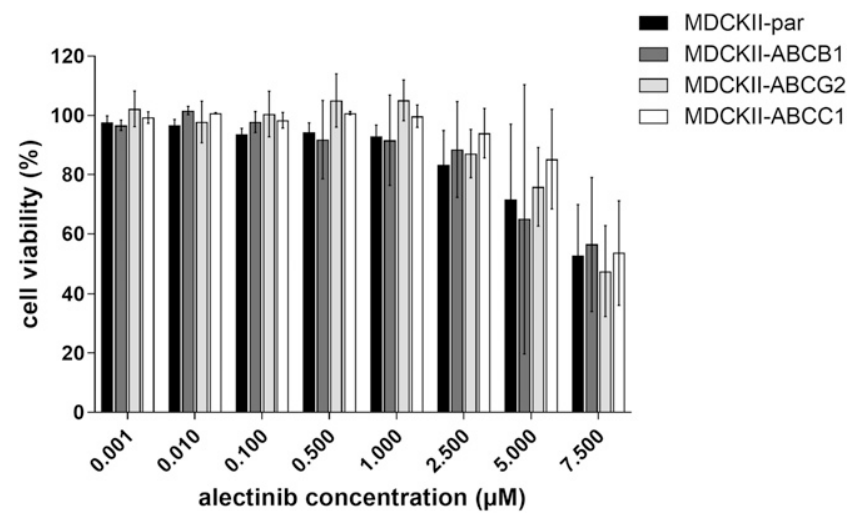

Fig. 7. Effects of $\mathrm{ABCB} 1, \mathrm{ABCG} 2$, and $\mathrm{ABCC} 1$ on cellular response to alectinib. The cells were exposed to alectinib and cell viabilities were determined after 48 hours of incubation using the MTT proliferation test. Due to limited solubility of alectinib, concentrations higher than $7.5 \mu \mathrm{M}$ could not be tested. Data were analyzed statistically using one-way ANOVA followed by Dunnett's post hoc test. ABC transporter-overexpressing cell viability values from each particular concentration point were compared with the corresponding viability values from parent cells, but no statistically significant changes were recorded for any of the points. Data are expressed as mean \pm S.D. from three independent experiments. 

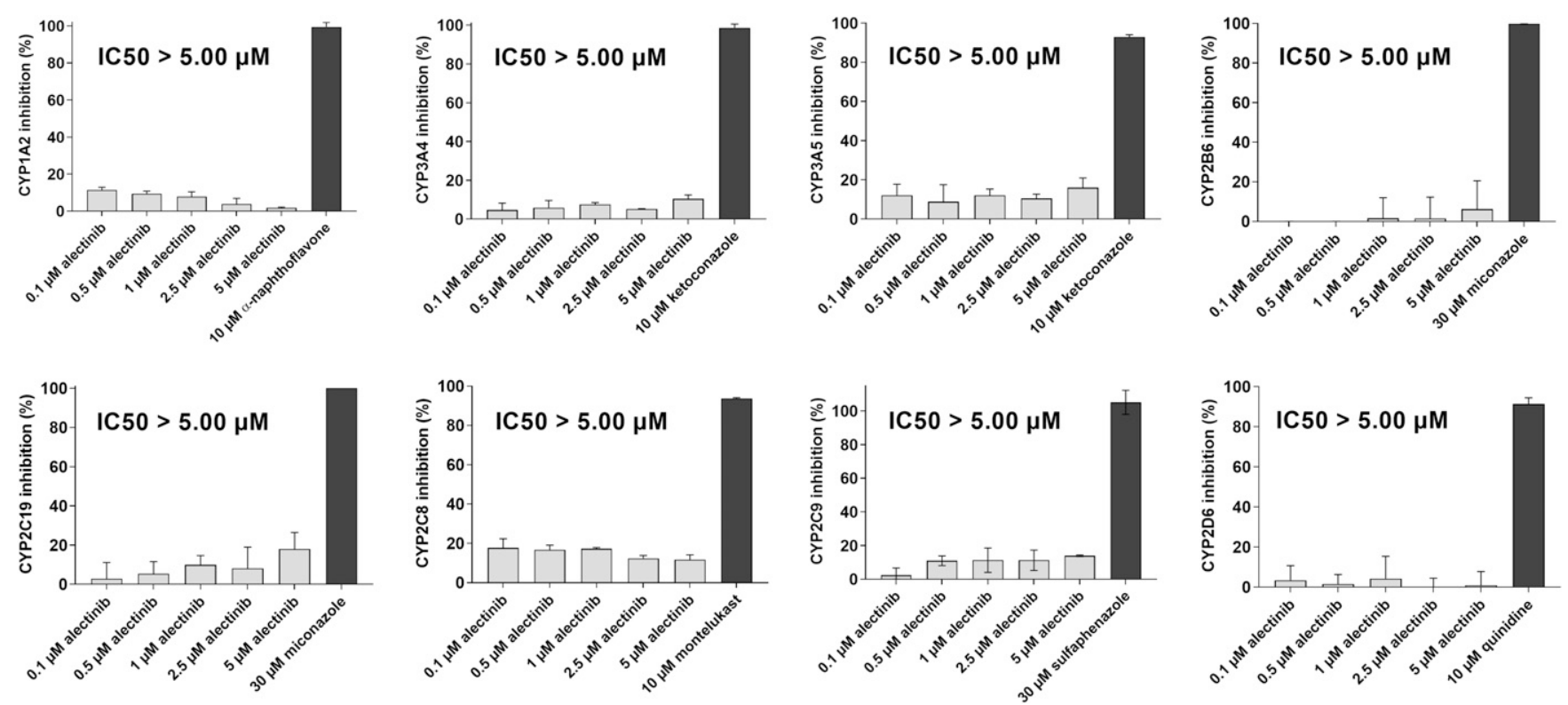

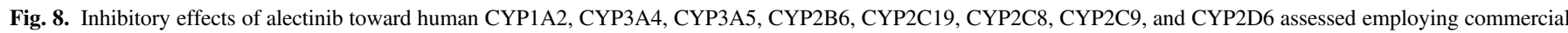

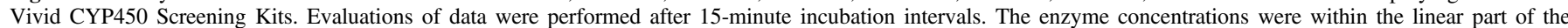

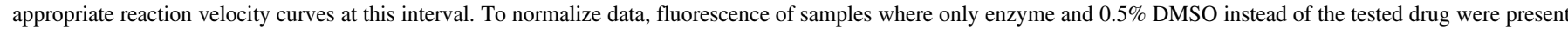

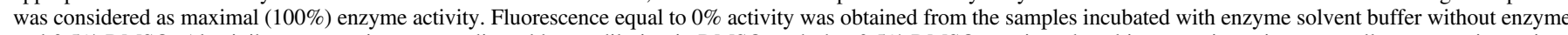

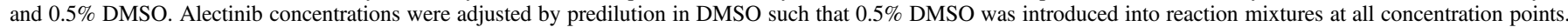
Data are expressed as mean \pm S.D. from three independent experiments.

results, it is possible to presume that the synergism observed between alectinib and $\mathrm{ABC}$ transporter cytotoxic substrates could allow for considerable dose reduction, which in turn would help to avoid the occurrence of such severe side effects as bradycardia; muscle, liver, lung, or kidney damage; myelosuppression; gastrointestinal or reproductive toxicity; and secondary carcinogenesis. In parental cell sublines, we also observed synergism between the examined drugs in certain parts of the $\mathrm{F}_{\mathrm{A}}$ range. Its level was nevertheless substantially less than seen in transporter-overexpressing cells. These results suggest that although $\mathrm{ABC}$ transporter inhibitory effects play the decisive role in the recorded MDR modulatory capabilities of alectinib, some additional pharmacodynamic and/or pharmacokinetic mechanisms presumably exist that contribute to the overall beneficial outcome of the tested drug combinations. Only limited information is available about synergistic combinations of alectinib with other anticancer drugs, but experimental data suggest MET or mTOR pathways to be the molecular targets to be hit concomitantly with the alectinib-inhibited ALK pathway to produce such an effect (Kogita et al., 2015; Redaelli et al., 2016). Similarly, in the clinical area, alectinib has not yet been approved in any combination for cancer therapy, and only three drug combinations are currently waiting to be evaluated in early clinical trials, namely, those including atezolizumab, cobimetinib, and bevacizumab [ClinicalTrials.gov (https:// clinicaltrials.gov/); identifiers NCT02013219, NCT03202940, and NCT02521051, respectively].

Apart from evaluating alectinib as a possible effective modulator, we aimed to look at the possible broadly opposite role of alectinib in pharmacokinetic MDR, which is to say whether its therapeutic effect could be compromised by this phenomenon. The results of MTT proliferation studies showed no significant changes in alectinib's antiproliferative effect between MDCKII-par and ABC transportertransduced MDCKII sublines, thus suggesting lack of impact on tumor response to alectinib from the presence of MDR-causing ABC transporters. These data correlate well with those reported by Kodama et al. (2014), who designated alectinib as an ABCB1 nonsubstrate using in vitro Caco-2 bidirectional transport assays and hypothesized that this fact constitutes a background principle for efficient penetration of alectinib into the brain. Subsequently, the absence of ABCB1 substrate properties of alectinib was indirectly confirmed in a study showing that ABCB1 overexpression is able to confer resistance to ceritinib but not to alectinib (Katayama et al., 2015). In contrast, no detailed published information is available regarding alectinib substrate affinity to either ABCG2 or ABCC1 or their roles in the development of resistance toward alectinib. In light of our results, alectinib shows ideal characteristics as an MDR reversal agent whose modulatory properties are not compromised due to efflux by any of the MDR-associated transporters. This limitation in being both a high-affinity transporter inhibitor and substrate is rather often seen for other TKIs acting as dual-mechanism drugs (Deng et al., 2014).

Along with $\mathrm{ABC}$ transporters, biotransformation enzymes represent additional causative factors participating in the development of pharmacokinetic MDR. To describe the possible role of alectinib in enzyme-meditated MDR, we further evaluated its interactions with CYP3A4, CYP3A5, and CYP2C8, which have been linked to decreased efficacy of paclitaxel, docetaxel, and vincristine in tumors. At the same time, these enzymes play predominant roles in pharmacokinetic drug-drug interactions together with CYP1A2, CYP2B6, CYP2C9, CYP2C19, and CYP2D6. To also cover this issue, in our study we included all of these eight $\mathrm{P} 450$ s that have been recommended by US Food and Drug Administration and EMA to be tested for inhibition (European Medicines Agency, 2012; Food and Drug Administration, 2017). Alectinib inhibited none of the enzymes tested at clinically relevant concentrations and thus lacks the potential for modulation of enzyme-mediated MDR as well as for acting as a perpetrator of clinically relevant DDIs. It has previously been reported that alectinib is mainly metabolized by cytochrome CYP3A to a major similarly active metabolite, M4 (Morcos et al., 2017; Nakagawa et al., 2018). In addition, alectinib has been described as a weak inhibitor of 
A

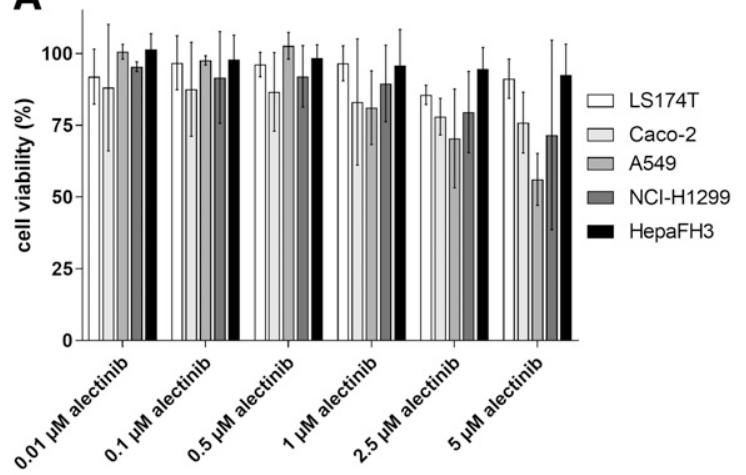

C

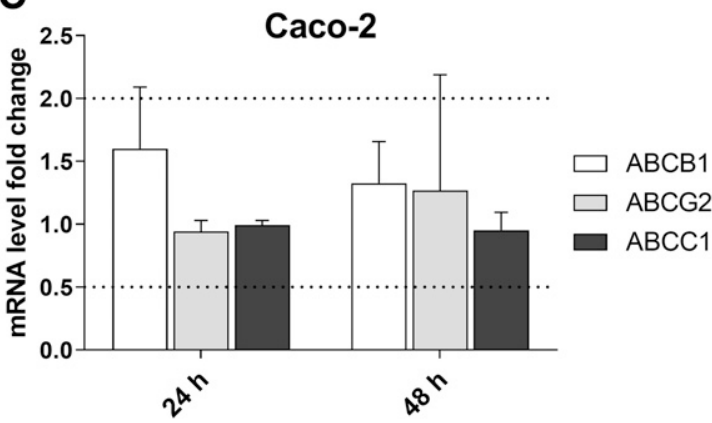

$\mathbf{E}$

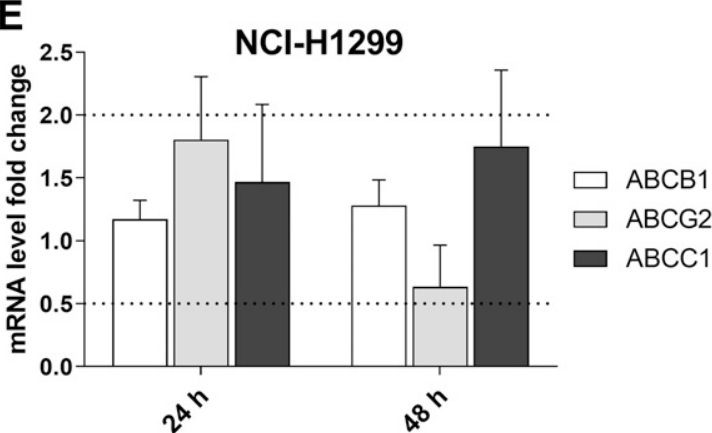

B

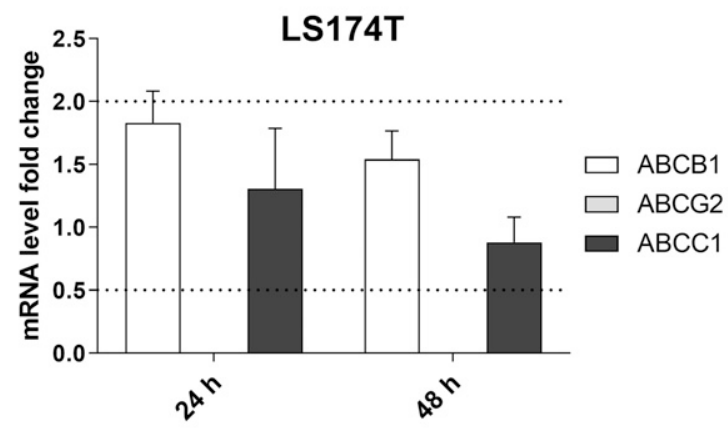

D

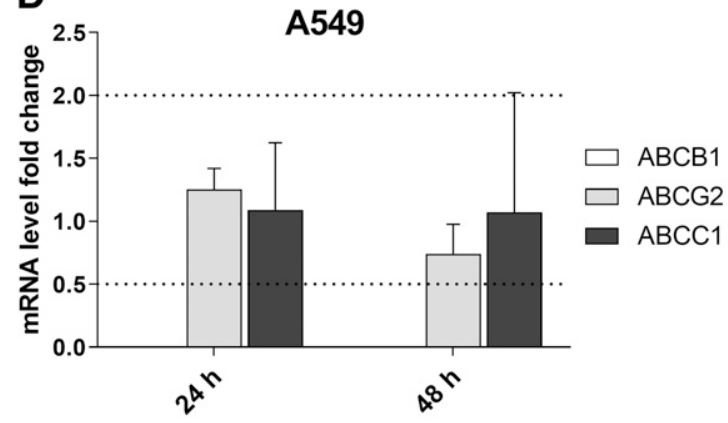

$\mathbf{F}$

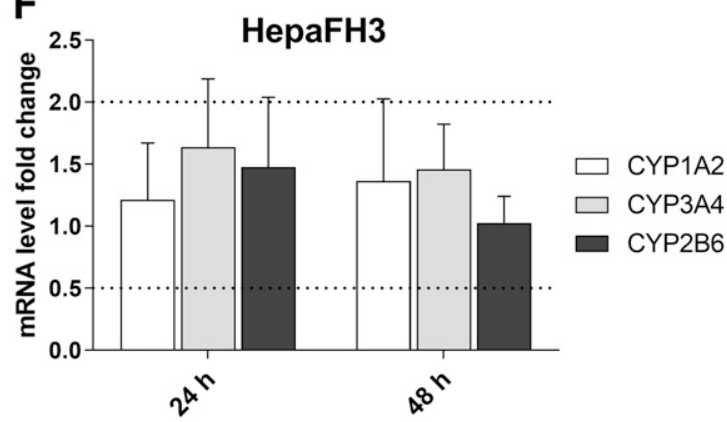

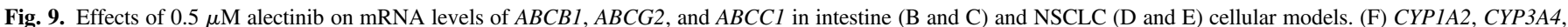

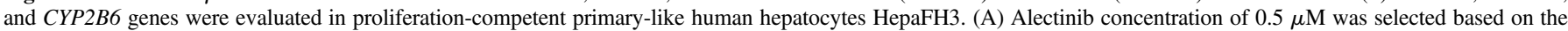

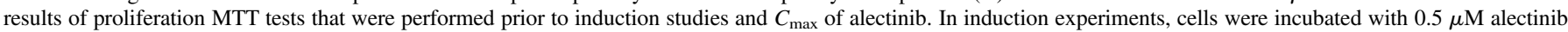

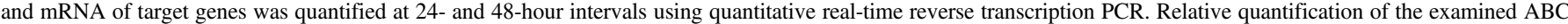

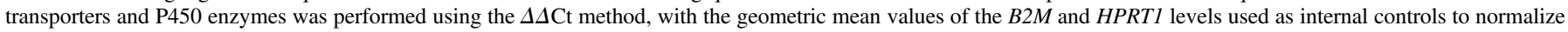
the variability in expression levels. Data are expressed as mean \pm S.D. from three experiments.

CYP3A, but alectinib has not been confirmed as either a victim or perpetrator by three in vivo drug-drug interaction studies involving healthy subjects and cancer patients (Morcos et al., 2017).

Apart from the inhibition, the induction of $\mathrm{ABC}$ transporters and $\mathrm{P} 450$ s by alectinib could constitute another phenomenon that is able to affect the pharmacological fate of concomitantly administered drugs. To address this possibility, we performed gene expression studies focused on $A B C B 1, A B C G 2$, and $A B C C 1$ and $C Y P 1 A 2, C Y P 3 A 4$, and $C Y P 2 B 6$ in suitable intestine (LS174T and Caco-2) and hepatic (HepaFH3) models, respectively. In addition, possible changes in $A B C B 1, A B C G 2$, and $A B C C 1$ gene expressions following exposure to alectinib were assessed in NSCLC cellular models (A549 and NCI-H1299) to reveal whether this TKI could affect the MDR phenotype of its target cancer cells. Alectinib did not provoke gene expression changes that would fit the criteria given by EMA for induction or downregulation (European Medicines Agency, 2012), and therefore its potential to become a perpetrator of induction-based DDIs as well as to influence the MDR phenotype of its target cancer cells can be considered low. No published information is available about induction potential of alectinib in relation to $\mathrm{ABC}$ drug efflux transporters. Regarding P450s, it was recently reported that alectinib is able to weakly induce CYP3A4 in vitro but that this phenomenon does not translate into any clinically meaningful effects, as observed by an in vivo DDI study with midazolam (Morcos et al., 2017).

In summary, we provide clear evidence that alectinib is an inhibitor of $\mathrm{ABCB} 1$ and $\mathrm{ABCG} 2$ but not of the $\mathrm{ABCC} 1$ transporter, and that it is able to effectively modulate transporter-mediated pharmacokinetic cytostatic resistance in a synergistic manner at clinically relevant concentrations. Importantly, alectinib shows ideal characteristics as an MDR reversal agent whose modulatory properties are not compromised due to efflux by any MDR-associated transporters. Furthermore, this TKI drug shows negligible potential to cause changes in the activities of P450 enzymes as well as in the gene expression levels of the examined P450s and ABC transporters. Our 
in vitro observations might serve as a valuable basis for future in vivo studies that could support the rational for our conclusions and possibly enable providing more efficient and safer therapy to many oncological patients.

\section{Authorship Contributions}

Participated in research design: Hofman, Ceckova, Staud.

Conducted experiments: Hofman, Sorf, Vagiannis, Sucha, Novotna.

Contributed new reagents or analytic tools: Kammerer, Küpper.

Performed data analysis: Hofman, Sorf, Vagiannis, Sucha, Novotna.

Wrote or contributed to the writing of the manuscript: Hofman, Sorf,

Vagiannis, Sucha, Novotna, Kammerer, Küpper, Ceckova, Staud.

\section{References}

Bayat Mokhtari R, Homayouni TS, Baluch N, Morgatskaya E, Kumar S, Das B, and Yeger H (2017) Combination therapy in combating cancer. Oncotarget 8:38022-38043.

Beretta GL, Cassinelli G, Pennati M, Zuco V, and Gatti L (2017) Overcoming ABC transportermediated multidrug resistance: the dual role of tyrosine kinase inhibitors as multitargeting agents. Eur J Med Chem 142:271-289.

Bhatnagar P, Wickramasinghe K, Williams J, Rayner M, and Townsend N (2015) The epidemiology of cardiovascular disease in the UK 2014. Heart 101:1182-1189.

Bienert S, Waterhouse A, de Beer TAP, Tauriello G, Studer G, Bordoli L, and Schwede T (2017) The SWISS-MODEL Repository-new features and functionality. Nucleic Acids Res 45 D313-D319.

Bugde P, Biswas R, Merien F, Lu J, Liu DX, Chen M, Zhou S, and Li Y (2017) The therapeutic potential of targeting $\mathrm{ABC}$ transporters to combat multi-drug resistance. Expert Opin Ther Targets 21:511-530.

Chou TC (2006) Theoretical basis, experimental design, and computerized simulation of synergism and antagonism in drug combination studies. Pharmacol Rev 58:621-681.

Cihalova D, Ceckova M, Kucera R, Klimes J, and Staud F (2015a) Dinaciclib, a cyclin-dependent kinase inhibitor, is a substrate of human ABCB1 and ABCG2 and an inhibitor of human ABCC1 in vitro. Biochem Pharmacol 98:465-472.

Cihalova D, Hofman J, Ceckova M, and Staud F (2013) Purvalanol A, olomoucine II and roscovitine inhibit $\mathrm{ABCB} 1$ transporter and synergistically potentiate cytotoxic effects of daunorubicin in vitro. PLoS One 8:e83467.

Cihalova D, Staud F, and Ceckova M (2015b) Interactions of cyclin-dependent kinase inhibitors AT-7519, flavopiridol and SNS-032 with ABCB1, ABCG2 and ABCC1 transporters and their potential to overcome multidrug resistance in vitro. Cancer Chemother Pharmacol 76:105-116.

Deng J, Shao J, Markowitz JS, and An G (2014) ABC transporters in multi-drug resistance and ADME-Tox of small molecule tyrosine kinase inhibitors. Pharm Res 31:2237-2255.

DeVita VT Jr and Chu E (2008) A history of cancer chemotherapy. Cancer Res 68:8643-8653.

Elkind NB, Szentpétery Z, Apáti A, Ozvegy-Laczka C, Várady G, Ujhelly O, Szabó K, Homolya L, Váradi A, Buday L, et al. (2005) Multidrug transporter ABCG2 prevents tumor cell death induced by the epidermal growth factor receptor inhibitor Iressa (ZD1839, Gefitinib). Cancer Res 65:1770-1777.

European Medicines Agency (2012) Guideline on the investigation of drug interactions. June 21, 2012 CPMP/EWP/560/95/Rev. 1 Corr. 2**. https://www.ema.europa.eu/en/documents/ scientific-guideline/guideline-investigation-drug-interactions_en.pdf

Ferreira RJ, Ferreira MJ, and dos Santos DJ (2013) Molecular docking characterizes substratebinding sites and efflux modulation mechanisms within P-glycoprotein. J Chem Inf Model 53: $1747-1760$.

Fletcher JI, Haber M, Henderson MJ, and Norris MD (2010) ABC transporters in cancer: more than just drug efflux pumps. Nat Rev Cancer 10:147-156.

Food and Drug Administration (2017) In Vitro Metabolism- and Transporter-Mediated DrugDrug Interaction Studies Guidance for Industry, Food and Drug Administration, Silver Spring, MD.

Giacomini KM, Huang SM, Tweedie DJ, Benet LZ, Brouwer KL, Chu X, Dahlin A, Evers R, Fischer V, Hillgren KM, et al.; International Transporter Consortium (2010) Membrane transporters in drug development. Nat Rev Drug Discov 9:215-236.

Guex N, Peitsch MC, and Schwede T (2009) Automated comparative protein structure modeling with SWISS-MODEL and Swiss-PdbViewer: a historical perspective. Electrophoresis 30 (Suppl 1):S162-S173.

Herzog N, Hansen M, Miethbauer S, Schmidtke KU, Anderer U, Lupp A, Sperling S, Seehofer D, Damm G, Scheibner K, et al. (2016) Primary-like human hepatocytes genetically engineered to obtain proliferation competence display hepatic differentiation characteristics in monolayer and organotypical spheroid cultures. Cell Biol Int 40:341-353.

Hofman J, Ahmadimoghaddam D, Hahnova L, Pavek P, Ceckova M, and Staud F (2012) Olomoucine II and purvalanol A inhibit ABCG2 transporter in vitro and in situ and synergistically potentiate cytostatic effect of mitoxantrone. Pharmacol Res 65:312-319.

Irwin JJ, Sterling T, Mysinger MM, Bolstad ES, and Coleman RG (2012) ZINC: a free tool to discover chemistry for biology. $J$ Chem Inf Model 52:1757-1768.

Jackson SM, Manolaridis I, Kowal J, Zechner M, Taylor NMI, Bause M, Bauer S, Bartholomaeus R, Bernhardt G, Koenig B, et al. (2018) Structural basis of small-molecule inhibition of human multidrug transporter ABCG2. Nat Struct Mol Biol 25:333-340.
Katayama R, Sakashita T, Yanagitani N, Ninomiya H, Horiike A, Friboulet L, Gainor JF, Motoi N, Dobashi A, Sakata S, et al. (2015) P-glycoprotein mediates ceritinib resistance in anaplastic lymphoma kinase-rearranged non-small cell lung cancer. EBioMedicine 3:54-66.

Kathawala RJ, Gupta P, Ashby CR Jr, and Chen ZS (2015) The modulation of ABC transportermediated multidrug resistance in cancer: a review of the past decade. Drug Resist Updat 18 $1-17$.

Kim Y and Chen J (2018) Molecular structure of human P-glycoprotein in the ATP-bound, outward-facing conformation. Science 359:915-919.

Kodama T, Hasegawa M, Takanashi K, Sakurai Y, Kondoh O, and Sakamoto H (2014) Antitumor activity of the selective ALK inhibitor alectinib in models of intracranial metastases. Cancer Chemother Pharmacol 74:1023-1028.

Kogita A, Togashi Y, Hayashi H, Banno E, Terashima M, De Velasco MA, Sakai K, Fujita Y, Tomida S, Takeyama Y, et al. (2015) Activated MET acts as a salvage signal after treatment with alectinib, a selective ALK inhibitor, in ALK-positive non-small cell lung cancer. Int J Oncol 46: $1025-1030$.

Li J, Jaimes KF, and Aller SG (2014) Refined structures of mouse P-glycoprotein. Protein Sci 23: $34-46$.

Manolaridis I, Jackson SM, Taylor NMI, Kowal J, Stahlberg H, and Locher KP (2018) Cryo-EM structures of a human ABCG2 mutant trapped in ATP-bound and substrate-bound states. Nature 563:426-430.

Michael M and Doherty MM (2005) Tumoral drug metabolism: overview and its implications for cancer therapy. J Clin Oncol 23:205-229.

Morcos PN, Cleary Y, Guerini E, Dall G, Bogman K, De Petris L, Viteri S, Bordogna W, Yu L, Martin-Facklam M, et al. (2017) Clinical drug-drug interactions through cytochrome P450 3A (CYP3A) for the selective ALK inhibitor alectinib. Clin Pharmacol Drug Dev 6:280-291.

Morris GM, Huey R, Lindstrom W, Sanner MF, Belew RK, Goodsell DS, and Olson AJ (2009) AutoDock4 and AutoDockTools4: automated docking with selective receptor flexibility. $J$ Comput Chem 30:2785-2791.

Muller IB, de Langen AJ, Giovannetti E, and Peters GJ (2017) Anaplastic lymphoma kinase inhibition in metastatic non-small cell lung cancer: clinical impact of alectinib. OncoTargets Ther 10:4535-4541.

Nakagawa T, Fowler S, Takanashi K, Youdim K, Yamauchi T, Kawashima K, Sato-Nakai M, Yu L, and Ishigai M (2018) In vitro metabolism of alectinib, a novel potent ALK inhibitor, in human: contribution of CYP3A enzymes. Xenobiotica 48:546-554

Prueksaritanont T, Chu X, Gibson C, Cui D, Yee KL, Ballard J, Cabalu T, and Hochman J (2013) Drug-drug interaction studies: regulatory guidance and an industry perspective. AAPS J 15: 629-645.

Redaelli S, Ceccon M, Antolini L, Rigolio R, Pirola A, Peronaci M, Gambacorti-Passerini C, and Mologni L (2016) Synergistic activity of ALK and mTOR inhibitors for the treatment of NPM-ALK positive lymphoma. Oncotarget 7:72886-72897.

Robey RW, Pluchino KM, Hall MD, Fojo AT, Bates SE, and Gottesman MM (2018) Revisiting the role of $\mathrm{ABC}$ transporters in multidrug-resistant cancer. Nat Rev Cancer 18:452-464.

Rochat B (2009) Importance of influx and efflux systems and xenobiotic metabolizing enzymes in intratumoral disposition of anticancer agents. Curr Cancer Drug Targets 9: 652-674

Siegel RL, Miller KD, and Jemal A (2018) Cancer statistics, 2018. CA Cancer J Clin 68:7-30.

Song Z, Wang M, and Zhang A (2015) Alectinib: a novel second generation anaplastic lymphoma kinase (ALK) inhibitor for overcoming clinically-acquired resistance. Acta Pharm $\operatorname{Sin} B \mathbf{5}$ : $34-37$.

Sorf A, Hofman J, Kučera R, Staud F, and Ceckova M (2018) Ribociclib shows potential for pharmacokinetic drug-drug interactions being a substrate of $\mathrm{ABCB} 1$ and potent inhibitor of $\mathrm{ABCB} 1, \mathrm{ABCG} 2$ and CYP450 isoforms in vitro. Biochem Pharmacol 154:10-17.

Szakács G, Váradi A, Ozvegy-Laczka C, and Sarkadi B (2008) The role of ABC transporters in drug absorption, distribution, metabolism, excretion and toxicity (ADME-Tox). Drug Discov Today 13:379-393.

Trott $\mathrm{O}$ and Olson AJ (2010) AutoDock Vina: improving the speed and accuracy of docking with a new scoring function, efficient optimization, and multithreading. J Comput Chem 31:455-461.

UniProt Consortium (2019) UniProt: a worldwide hub of protein knowledge. Nucleic Acids Res 47: D506-D515

Vadlapatla RK, Vadlapudi AD, Pal D, and Mitra AK (2013) Mechanisms of drug resistance in cancer chemotherapy: coordinated role and regulation of efflux transporters and metabolizing enzymes. Curr Pharm Des 19:7126-7140.

Waterhouse A, Bertoni M, Bienert S, Studer G, Tauriello G, Gumienny R, Heer FT, de Beer TAP, Rempfer C, Bordoli L, et al. (2018) SWISS-MODEL: homology modelling of protein structures and complexes. Nucleic Acids Res 46:W296-W303.

Wienkers LC and Heath TG (2005) Predicting in vivo drug interactions from in vitro drug discovery data. Nat Rev Drug Discov 4:825-833.

Yang K, Chen Y, To KK, Wang F, Li D, Chen L, and Fu L (2017) Alectinib (CH5424802) antagonizes $\mathrm{ABCB} 1$ - and $\mathrm{ABCG} 2-$ mediated multidrug resistance in vitro, in vivo and ex vivo. Exp Mol Med 49:e303.

Zhu V and Ou SH (2017) Safety of alectinib for the treatment of metastatic ALK-rearranged nonsmall cell lung cancer. Expert Opin Drug Saf 16:509-514.

Address correspondence to: Dr. Jakub Hofman, Department of Pharmacology and Toxicology, Faculty of Pharmacy in Hradec Kralove, Charles University, Akademika Heyrovskeho 1203, 50005 Hradec Kralove, Czech Republic. E-mail: jakub.hofman@faf.cuni.cz 